\title{
Review and Classification of Barriers and Enablers of Demand Response in the Smart Grid
}

DOI:

10.1016/j.rser.2017.01.043

\section{Document Version}

Accepted author manuscript

Link to publication record in Manchester Research Explorer

\section{Citation for published version (APA):}

Good, N., Ellis, K., \& Mancarella,P. (2017). Review and Classification of Barriers and Enablers of Demand

Response in the Smart Grid. Renewable \& Sustainable Energy Reviews. https://doi.org/10.1016/j.rser.2017.01.043

\section{Published in:}

Renewable \& Sustainable Energy Reviews

\section{Citing this paper}

Please note that where the full-text provided on Manchester Research Explorer is the Author Accepted Manuscript or Proof version this may differ from the final Published version. If citing, it is advised that you check and use the publisher's definitive version.

\section{General rights}

Copyright and moral rights for the publications made accessible in the Research Explorer are retained by the authors and/or other copyright owners and it is a condition of accessing publications that users recognise and abide by the legal requirements associated with these rights.

\section{Takedown policy}

If you believe that this document breaches copyright please refer to the University of Manchester's Takedown Procedures [http://man.ac.uk/04Y6Bo] or contact uml.scholarlycommunications@manchester.ac.uk providing relevant details, so we can investigate your claim.

\section{OPEN ACCESS}




\title{
Review and Classification of Barriers and Enablers of Demand Response in the Smart Grid
}

\author{
Nicholas Good ${ }^{1 *}$, Keith A. Ellis ${ }^{2}$, and Pierluigi Mancarella ${ }^{1}$ \\ ${ }^{1}$ School of Electrical and Electronic Engineering, University of Manchester, Ferranti Building, M13 9PL, \\ Manchester, UK \\ ${ }^{2}$ IoT Systems Research Lab, Intel Labs, Intel Corporation, Collinstown Industrial Park, Leixlip, County Kildare, \\ Ireland.
}

nicholas.good@manchester.ac.uk, keith.a.ellis@intel.com,p.mancarella@manchester.ac.uk

*Corresponding author Tel: +44 (0)161 3064807

\begin{abstract}
Demand for flexibility in electricity systems and the transition to the Smart Grid is increasing opportunities for demand response (DR). However, there are many barriers which prevent the full potential of DR being realised. Unlocking of this potential, through identification of DR enablers, can be aided through systematic classification and analysis of DR barriers. To this end, while previous works mostly focused on individual aspects, this paper develops a comprehensive 'socio-techno-economic' review, classification and analysis of DR barriers and enablers in a Smart Grid context. This provides an intellectual framework which may be used to underpin further work on the study and integration of DR. DR barriers are classified as either fundamental (i.e., relating to intrinsic human nature/essential enabling technology) or secondary (i.e., relating to anthropogenic institutions/or system feedbacks). Fundamental barriers are defined as economic, social or technological, whilst secondary barriers relate to political regulatory aspects, design of markets, physical (electrical network) issues, or to general understanding of DR. Subsequently, associated enablers for the defined barriers are suggested. Consideration of technical and commercial/social aspects for both power system and information and communication technology (the "internet of things") domains provides a foundational contribution to improve understanding of DR within the Smart Grid paradigm. Finally, the complexity resulting from connections between various barriers, enablers and the energy system generally, and the existence of the signature characteristics of complex systems is acknowledged and implications discussed.
\end{abstract}

Keywords: demand response, demand side management, smart grid, internet of things (IoT), flexibility, low carbon energy systems

\section{Introduction}

The need for increased flexibility in modern, low carbon electricity systems to maintain economic and secure operation has been well-documented [1,2]. In this respect, demand response (DR) is often considered a particularly suitable source of such flexibility, and one of the main components of the Smart Grid [3]. DR may be described as change in electrical energy usage by end-use customers from their normal consumption patterns, in response to some signal [4], typically an economic one, but not necessarily (e.g., it might be an 
environmental signal [5]). It may be based on direct/explicit control, or on indirect control via a price signal [6]. Directly controlled DR is typically used for system/network issues as reliability and speed of response is paramount in these situations. Price-activated DR is typically used in energy markets [4]. In the existing literature, the benefits of DR have been recognised in relation to the increased efficiency in grid and generation investment and in operation efficiency, particularly in systems with high renewable/distributed energy integration $[7,8]$. At the same time, DR has been appreciated as attractive, due to prevalence of DR potential, in domestic, commercial and industrial premises [9-11], and the lack of necessary substantial additional investment. However, due to the highly distributed nature of DR, which is a structural characteristic of the developing Smart Grid, the intrinsic and complex relationship with (heterogeneous, unpredictable, complex) people, and the requirement for enabling technologies, there are significant barriers to the adoption of DR schemes. Identification of these barriers and associated enablers is key to identifying how to overcome them, and increase the prevalence of DR.

In the remainder of the paper the existing literature on the barriers to DR and related literature on the barriers to energy efficiency (EE) is reviewed and the contribution of this paper is outlined in Section 2. Then, the fundamental and secondary DR barriers are classified and analysed in Section 3, before possible DR enablers are detailed in Section 4. Subsequently, in Section 5, the described barriers and enablers are summarised and the relationship between described barriers and enablers is discussed. Finally, in Section 6, conclusions from the work and resulting policy recommendations are detailed.

\section{Existing literature}

\subsection{Barriers to demand response}

There are several works in the literature addressing the issues/challenges/barriers related to DR. An early work covering challenges/barriers to DR is [7]. This work noted several key challenges to DR adoption. One was the lack of information and communication technology (ICT) infrastructure. Whilst this may be considered decreasingly relevant as developments in computing and Smart Grid ICT technologies enable DR [12], deficiencies in sensing, computing and communication/actuation for DR can be expected to hamper deployment for some time. Other barriers relate to the inter-related challenges of lack of understanding of DR and its system value, general complexity and the lack of appropriate market structures for realising the value of DR. The lack of appropriate market structures was also highlighted as a barrier in [13], in which barriers were regarded mostly as products of the required but unrealised changes to relevant institutions (such as markets). Such institutions are usually slow to adapt to advancements in areas such as aggregator functions [14] and communication infrastructure [15], which render restrictive rules on minimum unit size and telemetry less relevant. Kim and Shcherbakova offered some fresh perspective through the recognition of behavioural and informational consumer-related barriers, highlighting the often under-appreciated importance of the energy consumer, as a central actor in DR provision [16]. Most recently O'Connell et al. and Nolan and O'Malley offered comprehensive reviews of the issues around DR including analysis of challenges/barriers $[8,17]$. Key contributions here, with respect to previous work, are the foci on challenges related to DR markets, behaviour and business cases. Reinforcing the importance of appropriate market structures O'Connell et al. also highlighted the lack of market mechanisms, and regulation which prevented cost reflective market prices being passed through to the consumer [8]. A particular issue is agreement on how DR can be measured, and hence remunerated, i.e., what should be the baseline for any DR action [17]. Lack of market mechanisms, together with understanding of the potential value of DR, is further highlighted as a barrier to DR [17]. This can be understood as a lack of a business case, highlighted as a substantial barrier in its own right [18]. In addition to market/value barriers O'Connell et al. offers fresh and necessary perspective on previously underappreciated social elements, which is particularly relevant to DR from residential and small commercial consumers [8]. Specifically, O'Connell et al. highlights that consumers are economically rational to only a limited extent, and that various other priorities, such as comfort and convenience, can dictate behaviour [8]. 
This vein is furthered by Bradley et al. who define a framework of 'consumer' barriers based on findings from a UK pilot study [19]. Considering more physical aspects, the possibility of barriers related to network capacity have been highlighted, if DR synchronises demand (e.g., in response to a price signal) and results in loss of load diversity and violation of network capacity limits [20].

As demonstrated, the literature on DR, and the barriers (and, implicitly, enablers) for DR, is growing. In this literature technological aspects are appreciated but not systematically explored and market elements are central. However, a coherent and comprehensive classification of DR barriers, in particular one including analysis of social/behavioural aspects is missing. Such a classification may be informed, to some extent, by reference to the more developed literature on another important demand side measure, i.e., EE.

\subsection{Barriers to energy efficiency}

Given the variable nature of demand for, and the potentially multiple purchasers of, DR it is characterized by a dynamic and "smart" interaction with markets and by a greater role of Smart Grid technology. Although EE is not dynamic or "smart", the literature on EE may be useful to inform analysis of the more fundamental economic and social elements. An early work in the EE area [21] studied the 'paradox' of gradual diffusion of apparently cost-effective EE technologies. This work made the important observation, derived from the field classical economics, that 'barriers' could be categorised as market failures or non-market failures (alternatively, market barriers). In the first case the barrier is due to a failure of a market to operate properly. Thus the barrier can be removed by improving the functioning of the market. In the second, the barrier is due to non-(classical) economic reasons. Sorrell builds on this separation of barriers into market and non-market failures, by defining barriers as: (i) economic; (ii) behavioural; and (iii) organisational; although the typology is not exclusive, and barriers may have multiple and overlapping aspects [22]. Moving into literature on electricity demand reductions more generally, behavioural aspects are attracting further interest more recently. Chiming with the realisation of the importance of social aspects to DR, especially for residential and small commercial consumers [8], the uncertainty on consumer preferences (which are often time-variant and inconsistent; i.e., economically irrational) has been highlighted as a particularly intractable barrier to exploitation of DR [23-25]. Emerging from this literature, and also useful for DR, is therefore a broad classification of the fundamental barriers to EE as either economic (market failures and market barriers) or social (behavioural and organisational barriers). Though it should be highlighted that the complexity of the relevant systems means that such classes are interrelated [25].

\subsection{Contribution}

The contribution of this work is to address the lack of a systematic analysis of the 'fundamental', and derivative secondary, barriers to DR in the current literature on DR challenges/issues/barriers, which is crucial if the penetration of DR, which may be the most cost effective source of flexibility, is to be increased. As reasoned in section 3.1, fundamental barriers can be understood as barriers which relate to intrinsic human nature (social/economic barriers), and to essential enabling technology (technological barriers) in a Smart Grid context. Such barriers are relevant to DR from all sectors of electricity consumers (industrial, commercial and residential). These fundamental barriers (and associated enablers) cover power system and ICT technical and commercial/social aspects, ensuring full coverage of relevant perspectives of the Smart Grid vision. This comprehensive 'socio-techno-economic' classification and analysis provides an intellectual framework which covers the fundamental aspects of DR, on which analysis of specific DR schemes can be based. Recognising the importance of more practical DR barriers, secondary barriers are also examined. The broad classifications and hierarchy of DR barriers (and, hence, enablers) is demonstrated in Figure 1. This comprehensive classification may be used to underpin further work on the study and integration of DR, and thus be a useful contribution to the field. 


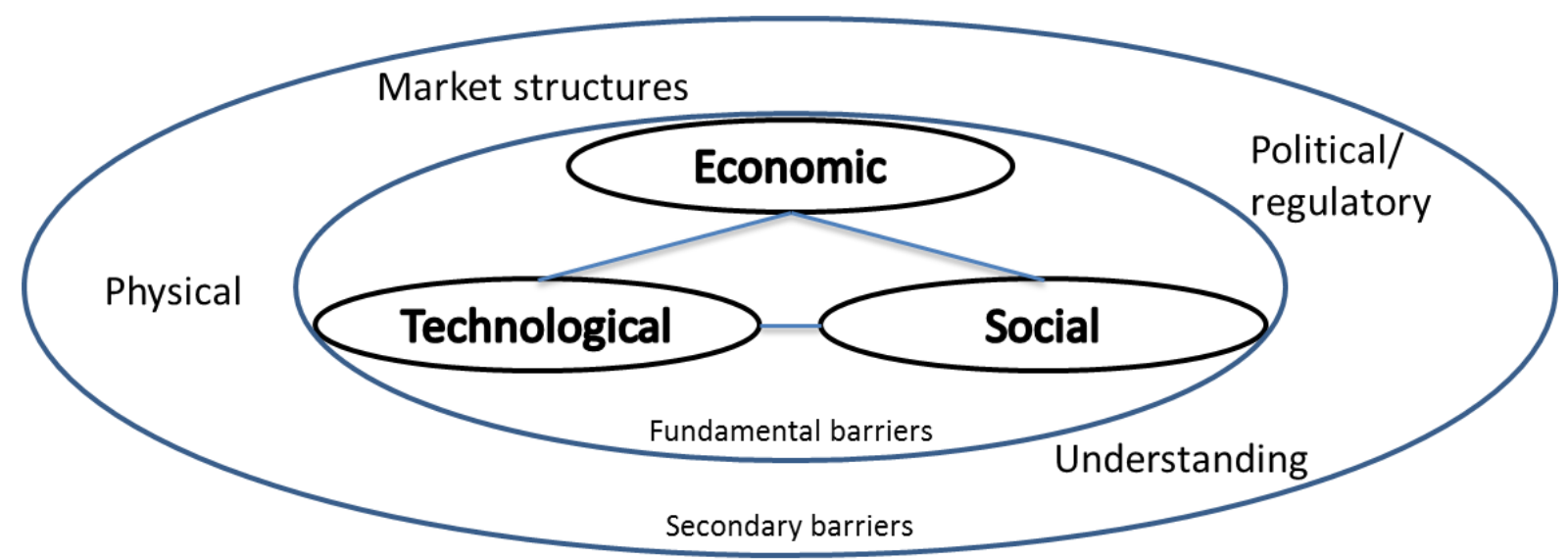

Figure 1: Classification and hierarchy of DR barriers

\section{Barriers to DR}

As noted in section 2.2, literature on EE defined barriers as economic, behavioural or organisational [26]. The common factor in theories which relate to these barrier classes is that they relate to how individuals (themselves or as components of organisations) interact with each other. This motivates the inclusion of economic and social (including behavioural and organisational) barriers as fundamental as DR, like EE, is ultimately based on the decisions made by individuals. In addition to economic and social barriers a technological barrier class should be defined and classed as fundamental, given that sensing, computation and communication are crucial to realisation of DR and the Smart Grid generally. All other barriers can then be defined as secondary. We consider barriers as secondary if they derive from fundamental economic, social or technological aspects. They can be understood as either relating to anthropogenic institutions (e.g., markets and regulation), or system feedbacks (e.g., electrical network constraints).

\subsection{Fundamental barriers to DR}

As aforementioned, 'fundamental' barriers are barriers relating to intrinsic human nature, or essential enabling technology. Without an understanding of these barriers, there is no chance of an optimal deployment of enabling interventions. Fundamental barriers can be classed as economic, social or technological, covering both power system and ICT perspectives of the Smart Grid. Below each class is explored and broken down to describe all relevant barriers.

\subsubsection{Economic}

Partly due to the existence of a convenient framework for their analysis (i.e., classical economic theory) there is a large body of work on economic barriers to EE, which can inform analysis of DR barriers. This work generally involves study of market failures (i.e., flaws in the way a market operates) and market barriers (i.e., other obstacles to the given objective) [21,26-30]. These classifications can be readily applied to Smart Grid DR markets.

\subsubsection{Market failures}

Drawing particularly on [26,28-30] Table 1 describes the three general classes of market failures relevant to DR, which are expanded upon below. These classes include some failures often presented separately. For example, as detailed below, the 'split incentives', 'adverse selection' and 'principal-agent problem' failures which are often separately presented are all information-related, and may thus be defined as particular cases of the imperfect information failure.

Table 1: Classification of market failures 


\begin{tabular}{|l|l|}
\hline Imperfect information & $\begin{array}{l}\text { Classical economics assumes that all parties have access to free and perfect } \\
\text { information. In reality this may not occur, which constitutes a failure. }\end{array}$ \\
\hline Incomplete markets & $\begin{array}{l}\text { Markets in which property rights are not well defined can be termed incomplete. } \\
\text { This is a failure as it can result in a discrepancy between private and social costs } \\
\text { and benefits [26]. }\end{array}$ \\
\hline Imperfect competition & $\begin{array}{l}\text { Uncompetitive markets, where one or more parties have, and exercise, market } \\
\text { power. }\end{array}$ \\
\hline
\end{tabular}

Imperfect information can occur for a number of reasons. Firstly, there is a substantial lack of knowledge, on the part of potential DR providers (e.g., domestic, commercial or industrial consumers), on their variable and uncertain consumption, and hence their potential to provide DR [16]. With respect to DR markets, there are (cash and time) costs associated with collecting and processing information [28]. These search costs form part of the transaction costs of partaking in a market. The existence of these costs means that it may not be costefficient for parties to collect all the relevant information, resulting in an 'Adverse selection' market failure, where one party to a trade is better informed than the other [26,29]. This is likely as the Smart Grid is very complex, which will increase search costs. Imperfect information may also arise if markets are so immature that the demand for certain types of information is not sufficient to motivate its collection and distribution by market participants [29]. Examples of imperfect information market failures could be when a small commercial business cannot predict its (flexible) electric low grade heating load, a potential DR provider finds accessing appropriate markets to expensive and/or time consuming, or when information on the effect of heat pump cycling on wear and tear (as would result from frequent DR calls) is not available.

A special type of information-related failure is that of asymmetric information, producing split incentives, where one party acts for another, but does not accurately reflect their interests $[26,28,29]$. If one party has access to information which it cannot effectively communicate to another party (e.g., due to large transaction costs), then the parties have split incentives which cannot be reconciled through a contract, because they are not defined. In the case of a DR provider who has contracted with an aggregator, this could arise if the aggregator cannot fully understand the nature of the DR resource, as it cannot understand the user preferences (e.g., relating to comfort) which dictate (at least some) of the flexibility. If such user preferences were fully understood, there would be no failure, as the terms for flexibility exploitation could be defined in a contract. This can be defined as the 'principal-agent problem' [26,29]. In this case the 'principal' (i.e., DR provider) does not have the necessary information (e.g., the value they assign to thermal comfort) to define a contractual obligation on the 'agent' (i.e., aggregator). This can lead to opportunistic behaviour on the part of the agent, which is not in the interest of the principal.

As detailed in [26], incomplete markets may arise when property rights are not well defined, i.e., comprehensively assigned, exclusive, transferable, and secure. For example, the costs of unregulated $\mathrm{CO}_{2}$ emission are not exclusive; such costs accrue to many parties through increased atmospheric warming, and associated implications. The existence of this 'externality' (a cost or benefit that affects a party who did not choose to incur it) constitutes a market failure, which can lead to increased levels of $\mathrm{CO}_{2}$ emission, with the associated increased cost to society. Another example of an incomplete market is where benefits of an asset are not excludable (i.e., the benefits of investment may, at least partially, accrue to third parties), such as can be the case with DR where a reduction in peak load may benefit many actors [7,31]. This can result in some parties free-riding (benefiting from a good, without paying for it), which is a clear market failure [32]. This may the case with DR, if DR bought by a retailer, to reduce imbalance, also benefits a distribution network operator, by relieving congestion This can also include indirect free-riding, such as when insufficiently costreflective imbalance penalties fail to penalise undesirable behaviour of DR providers or aggregators, resulting in the costs that are not recovered by the imbalance penalties being socialised (spread) over all system users [33]. 
Lastly, a clear market failure occurs when a party/parties have such a large market share that they are able to exert market power, creating imperfect competition. In this scenario, parties can charge prices in excess of their marginal costs, resulting in an inefficient market. This may occur if the near oligopoly of electricity supply, in many systems, is transferred to Smart Grid/DR markets [34].

\subsubsection{Market barriers}

As with the classes of market failures, there are many classes of market barriers. An extensive list is given in [30]. Many of those classes, however, relate to information or behaviour, and are thus covered by the 'imperfect information' market failure, or by behaviour-related barriers (see section 3.1.2.2). Drawing on the work of Thollander et al. and Chai and Yeo the remaining market barriers are summarised in Table 2 [29,30].

Table 2: Classification of market barriers

\begin{tabular}{|l|l|}
\hline Market barrier & Description \\
\hline Access to capital & $\begin{array}{l}\text { Some DR may require additional capital investment. For some parties, with little } \\
\text { reserves and/or poor credit rating, accessing capital may be problematic. }\end{array}$ \\
\hline Uncertainty & Uncertainty on future revenue/costs can pose a substantial barrier. \\
\hline Hidden costs & $\begin{array}{l}\text { Hidden costs related to market participation i.e., negotiation and enforcement } \\
\text { costs associated with transactions may be a barrier. }\end{array}$ \\
\hline $\begin{array}{l}\text { System value/ } \\
\text { Demand for DR }\end{array}$ & $\begin{array}{l}\text { It is possible that flexibility is simply not valued in a system. This can be a barrier } \\
\text { to DR. }\end{array}$ \\
\hline
\end{tabular}

The degree to which access to capital is a barrier to DR will vary depending on the degree of investment required (which may be small, if only enabling sensing and communication technology is required), and the party making the investment (given varying degrees of credit worthiness amongst potential investors). Inclusion of uncertainty as a separate market barrier is debatable. As highlighted in [29], it may be considered a barrier if parties are not able to reduce the implication of uncertainty to a calculated risk. This is highly likely, especially over the long term, given the uncertainty on the reaction of other parties, including regulators, to increased DR activity within the Smart Grid. For example, increased local generation in peak use-of-system charge periods may motivate a shift in use-of-system charges to a subscription (kW) based levy [35].

Hidden costs relate to the costs associated with participation in markets. These include negotiation and enforcement transaction costs (i.e., that costs relating to negotiating and enforcing contracts), but not search transaction costs (i.e., the costs of locating information on the opportunities for exchange), which relate to the imperfect information market failure [36]. If these hidden costs are excessive, they could represent a barrier to DR. Such costs can been 'outsourced' to organised markets, who will charge fees in return for providing access to many counterparties, with whom they may trade standardised products (eliminating negotiation costs), with a guarantee of delivery (eliminating enforcement costs). Such fees should be taken into account, as they may pose a barrier, particularly to small parties.

System value or market demand is not considered a barrier on the literature on EE, as the value of reducing energy consumption is assumed. However, for DR this is not so, as the value of flexibility is not axiomatic. Although all systems are likely to have some value for flexibility (e.g., for system balancing and reserve provision purposes), some systems will have more value than others. For example, flexibility may be more valuable in systems with highly variable and unreliable generation, i.e., wind and solar, as such systems are more uncertain and unstable, due to the uncertainty of renewable generation and the reduced amount of committed synchronous generation, and require increased frequency response capability and reserves [37]. This lack of value implies a lack of market demand for flexibility, but it should be emphasised that the reverse is not necessarily true. Illustrating the complexity of energy systems and DR markets, as explored further in section 5, lack of market demand may result from other factors besides a lack of inherent value. For example, social barriers may suppress interest from potential providers, technological barriers may make provision of DR too inconvenient, regulatory barriers may impose onerous requirements or market structure barriers may 
prevent DR products from reflecting the true value of flexibility. Returning to consider system value, it should be noted that as electricity systems evolve towards the Smart Grid the value of DR will also change. Notably, increased penetration of renewable energy resources will increase demand for system flexibility, whilst increased electricity load on distribution networks will increase demand for local flexibility, both of which may be provided by DR $[10,38,39]$.

\subsubsection{Social}

Social barriers may, in the first instance, be usefully classified, following [26], as organisational and behavioural. Organisational barriers may be relevant to commercial parties, as such barriers relate to the social systems of such structured organisations. However, arguably of greater importance for DR are behavioural barriers, given the high number of individual interactions which can affect DR provision.

\subsubsection{Organisational barriers}

Sorrell identifies two organisational barriers, namely, power and culture [26]. Power (or lack of it) may be a barrier where it relates to the power of the person within an organisation who has a responsibility for implementing a DR programme. If the relevant person does not wield enough power within their organisation, e.g., to install necessary enabling technology, to instruct (to the degree possible) behaviour change, or to invest in increased flexibility, this may form a barrier to DR. Power, as a barrier, is closely linked to the less precise barrier of organisational culture. Indeed insufficient power for the DR decision-maker is likely, in some part, to be due to the prevailing culture of the organisation. Specifically, if energy, environmental and even economic concerns (outside of the core business), are not generally regarded as important within the organisation, then this will form a general "soft" barrier to DR.

\subsubsection{Behavioural barriers}

Behavioural barriers may be described as those factors which explain why the behaviour of any individual deviates from that of the ideal, fully rational (in the classical economic sense) agent [40]. For a firm, rational means profit-maximising, whilst for an individual it means utility-maximising. This latter definition is more complex, as an individual must consider factors such as convenience and comfort, as well as cash. The behavioural barriers which can be relevant to DR are shown in Table 3.

Table 3: Behavioural barriers

\begin{tabular}{|l|l|}
\hline Barrier & Description \\
\hline $\begin{array}{l}\text { Form of } \\
\text { information }\end{array}$ & $\begin{array}{l}\text { If information is not regarded as intended by the sender, the corresponding } \\
\text { behaviour of the recipient will not as expected by the sender. }\end{array}$ \\
\hline $\begin{array}{l}\text { Credibility } \\
\text { and trust }\end{array}$ & $\begin{array}{l}\text { How the recipient of information regards the sender will dictate how such } \\
\text { information will be perceived. }\end{array}$ \\
\hline Values & $\begin{array}{l}\text { Besides cash cost minimisation, consumers may be influenced by their values (e.g., } \\
\text { environmental values, energy conservation values). This may prompt behaviour which } \\
\text { does not align well with DR. }\end{array}$ \\
\hline Inertia & $\begin{array}{l}\text { The entrenchment of behaviour may be a barrier; as such behaviour can take time to } \\
\text { change, even if there is clear benefit to doing so. }\end{array}$ \\
\hline $\begin{array}{l}\text { Bounded } \\
\text { rationality }\end{array}$ & $\begin{array}{l}\text { Cognitive capacity of an individual is naturally limited, which may mean that, even } \\
\text { with the necessary information, they may not reach the optimal DR-related decision. }\end{array}$ \\
\hline
\end{tabular}

Given the reliance of timely response of DR providers to DR signals, the form of information has the potential to be a significant barrier if it inhibits communication between DR buyers and providers. An example can be the design of the user interface, where poor design has been shown to result in unexpected behaviour [3].

Given the importance of interactions between DR providers and buyers, the credibility of the relevant parties and the level of trust between such parties are crucial. Low levels of credibility and trust can pose a barrier to DR. For example, do DR providers trust the sender? Do they perceive them as reliable? Do they identify with them (do they think the sender has the same values)? The issue of trust is identified as significant in 
acceptance of DR $[3,41]$. An example of the importance of trust and credibility could be found in the preference of DR providers to interact with smaller, local DR buyers, who they identify with and trust, rather than large, impersonal energy utilities. In particular, it can be linked to the reticence to allow third party control of devices. Behaviour in this area however is complex, given the influence of consumer values. Such values can inform user preferences with regard to concepts such as autonomy, ownership, power and control [42], especially ownership of personal information that may be derived from data [43]. This is particularly pertinent for DR given the large amount of communication of information required by DR, due to the use of open or untrusted networks and the potentially huge number of physically distributed devices, from multiple vendors. Hence consumer values may form a barrier to DR if 'anti-DR values', such as desire for autonomy, control, comfort etc. win out of possible 'pro-DR values', such as environmental and energy conservation values. As ever, the picture here is not straightforward, as the relationship between values and preferences is not always clear and consistent. For example, initially pro-DR behaviour can give way to anti-, or ambivalent behaviour over time as the cumulative time or exertion cost grows [16]. Further, the recognised 'value-action gap' phenomena can complicate efforts to encourage DR [24]. Consumer values may be particularly significant for domestic DR providers, who are likely to prioritise comfort and convenience. Degrading consumer comfort, or requiring significant interaction of residents with control signals, for example, may be a significant barrier to DR, and has been shown to be so [44].

Linked to the values barrier is the barrier of inertia. Consumers can be reluctant to change behaviour, even if there are clear benefits to doing so. This can manifest itself in focus on the perceived inconvenience of DR participation, and the requirement that interventions "fit" with current lives [45]. However, given the complexity of the energy, economic and social systems in which DR takes place, it has been posited that inertia of this kind may be a rational reaction to the bounded rationality of the consumer, economising on cognitive exertion by employing heuristics informed by past experience [46]. For example, a DR provider may not want to increase their flexibility by installing more energy storage, even if it is clear this will be profitable and the investment will pay back quickly. If understanding the business case is time-consuming or intellectually difficult, the DR provider may prefer to simply base their decision on the evidence of profitability in the recent past. Another result of bounded rationality can be non-optimal satisficing behaviour (e.g., in choosing whether to engage in DR to reduce overall energy costs, or not), in which consumers, overwhelmed by options, settle for 'good enough' [16]. Here it is worth highlighting that bounded rationality and inertia (as for all behavioural barriers, to a greater or lesser extent) can form barriers to DR from firms, as well as individuals. With firms, they may cause entrenched attachment to established business cases, obscuring the potential of new, Smart Grid/DR-related, business cases.

\subsubsection{Technological}

The centrality of technology to DR and the whole Smart Grid necessitates consideration of ICT aspects, in assessment of barriers to DR [47]. In the context of DR and the Smart Grid, in particular, the internet of things (IoT) may be used interchangeably with ICT.

ICT underpins local metering (to determine DR flexibility and delivery), transactional communications (between DR provider and purchaser) and on-premises automation (to enact DR) $[48,49]$. ICT that can enable DR has been readily available for some time, albeit DR services have been the preserve of large industrial loads. The issue that presents itself today is in moving from 'niche to normal' in delivering DR capability at scale beyond industrial scenarios [50].

At an abstract level technological barriers can be loosely considered as being sensing-related, computingrelated and communication-related. Standardisation is pertinent in all cases. Another related challenge, which relates to a more social aspect, pertains to the finding and retaining of technical talent. These challenges are outlined in the sections that follow. 


\subsubsection{Sensing}

The most basic type of sensing required for DR is electricity metering so much so the EC has mandated for the creation of a standardised technical architecture for same [51]. Energy markets trade in 15 minutes to 1 hour periods, requiring reliable metering at this resolution in order to participation in such markets. Barriers to DR may exist where the necessary metering infrastructure is not present, however, this is not a technological issue but rather an implementation issue relating to technology. Beyond utility/billing grade metering, participation in other explicit DR schemes, such as frequency response, reserve or constraint management, will most likely require metering at an even finer resolution.

Granular sensing is required to assist in identifying flexibility, to certify actuation and to measure related factors associated with delivered energy services, e.g., thermal comfort, appliance availability. Here, inadequate systems (e.g., building automation systems) may form a barrier to DR if they do not adequately highlight flexibility and capture the effect of DR on consumer experience e.g., comfort/convenience/cost. The importance of measurement, verification and impact for settlement are discussed in [52]. While the importance of achieving DR without impacting comfort is discussed in [53] and cold storage [54,55].

The primary issue relates to sensing at high frequency, with high reliability, but in a way that is flexible and extensible with respect to enabling additional, often yet to be envisaged, Smart Grid services and/or devices, and all at an acceptable cost. This poses a technical and standardisation challenge, especially regarding interoperability, because, while the primary sensors/actuators involved pertain to the electrical energy domain, the sensors involved in inferring the context required to identify flexibility and impact on users is varied. This is before one even considers the heterogeneity introduced by different vendors and protocols which will proliferate with the development of the Smart Grid. Standardisation is central and is further addressed in section 3.1.3.4.

\subsubsection{Computing}

Adequate sensing is necessary, but not sufficient, for optimal DR. Sensing can generate large amounts of data, whilst uncertainty, e.g., in the determinants of DR potential and in DR prices can increase the computational load of any stochastic/robust optimisation [56] that may be needed to effectively cope with uncertainty, especially when at scale. Whilst 'big data' technologies can deal with voluminous, heterogeneous, near-realtime and static data, and be used to identify and exploit new patterns within that data, there are limits, especially when decisions are critically time-constrained, e.g., frequency response, or where cloud services are not trusted (see section 3.1.3.3). Additionally, data scientists need to be able to work with domain experts and have a certain capacity for understanding the Smart Grid context, see section 3.1.3.5.

Computation can be linked to the social bounded rationality barrier (see section 3.1.2.2), whereby the cognitive capacity of an individual is naturally limited. This issue of bounded capacity can also be found in the computation domain. Edge devices (i.e. intelligent compute devices that are located locally to the physical assets being sensed and/or controlled), especially loT embedded devices, are likely to be compute constrained and therefore services may require greater compute capacity at certain times, now or into the future. This infers a requisite level of extensibility and flexibility, which in turn points to the need for connectivity, whereby additional (third-party) computation capability can be leveraged. Again trust plays a part here (see sections 3.1.2.2, 3.1.3.3), with data exchange and the standardisation of same arguably a bigger barrier than the computational capacity. Again, like sensing (see section 3.1.3.1), the primary issue relates to being able to deliver the right compute capacity, where and when required, and at an acceptable cost, while meeting security and privacy requirements.

\subsubsection{Communication}

Assuming accurate sensed data (see section 3.1.3.1), one must then deal with access to that data. Wired and wireless sensors/actuators need to connect to edge (local) compute and cloud compute (see section 3.1.3.2) in an agreed, standardised way. This standardisation must address data formatting, transfer, transformation and 
semantics. At a macro level, if data exchange could be agreed by all actors then the DR concept could be delivered in rapid time and at scale. However, in reality, interoperability has traditionally been, and continues to be, a very slow process taking many years due to competing approaches and alliances [57].

Figure 2 [58] gives a pertinent example illustrating the myriad of loT alliances that traverse the various communication layers and potential domain verticals. Similar issues are relevant with respect to data acquisition specifically for 'smart' metering. When faced with this level of purposed options it can very difficult to make any sort of investment decision, which acts as a barrier to adoption. Again standardisation is central, see section 3.1.3.4.

Central to communication are barriers pertaining to data security and privacy which all relate to imperfect information (see section 3.1.1.1) credibility and trust, see section 3.1.2.2. There are several features of loT systems particularly relevant to large scale systems supporting energy services, specifically relating to the tendency of such systems to be:

- $\quad$ physically distributed

- a mixture of very small to very large devices

- dependent on closed and open or untrusted networks

- large scale deployments, which may extend to tens of thousands of components

- $\quad$ different parts of the system may be created by different vendors

- $\quad$ use and functionality changes over the duration of the system's lifecycle

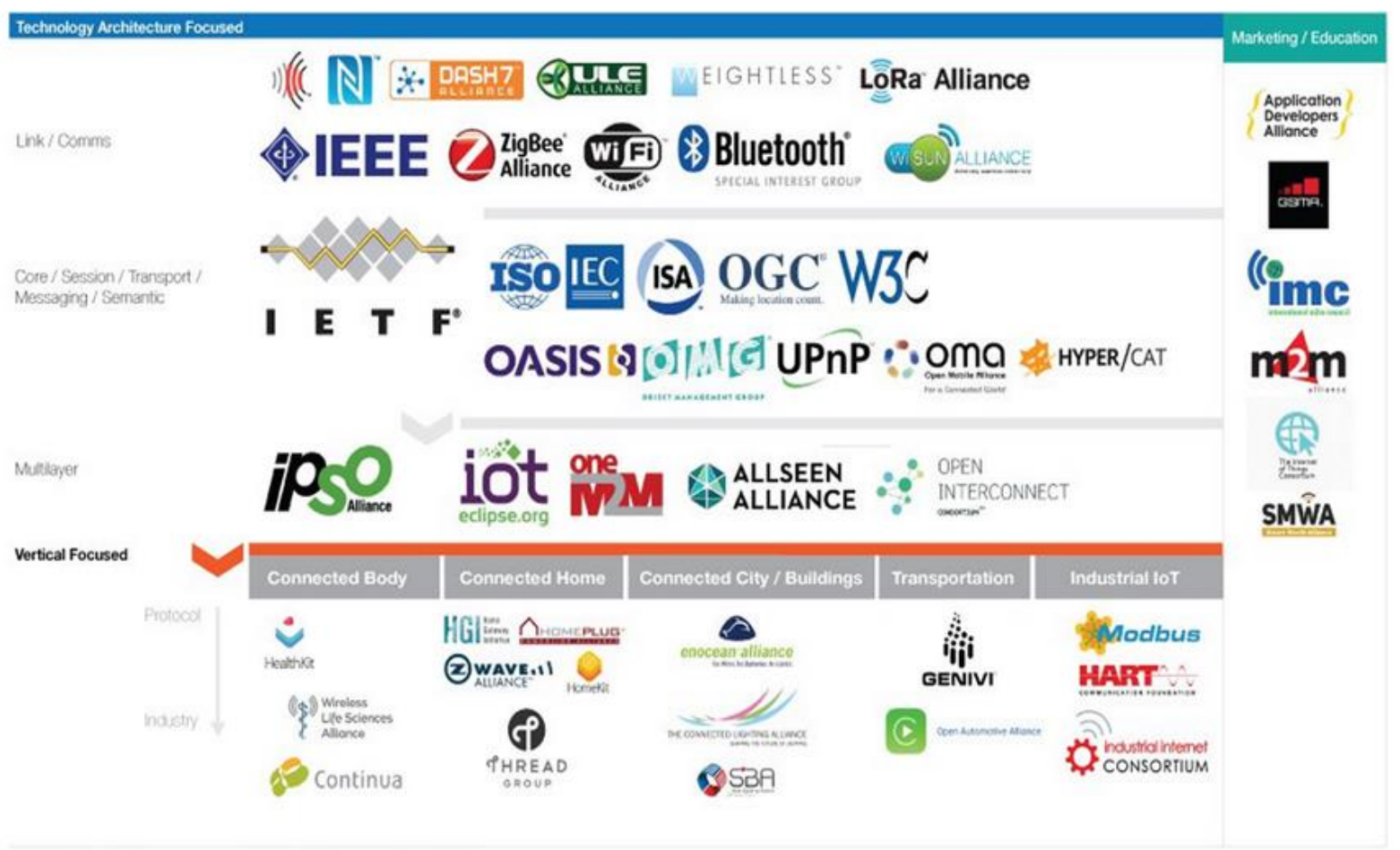

Figure 2: IoT Alliances round-up [58]

If not addressed, security concerns can act as a considerable barrier to adoption, especially with cloud based technologies that require storage and processing of data off-premises. In an EC/IDC analysis of the demand of cloud computing services in Europe and barriers to uptake, Figure 3 [59], 62.2\% of all respondents identified the top six concerns listed as being either a very large or complete barrier to cloud adoption, with the top five directly or indirectly related to security or privacy. This arguably can be considered indicative of the wider importance of such matters on loT adoption required for DR. 
Separate to security is the issue of data privacy. As outlined in section 3.1.2.2, unwillingness to share data due to fears of misuse or profiteering is a very real barrier to $D R$, or more specifically to the adoption of the technology that underpins it. Developing loT solutions that do not account for these concerns can impact the adoption/investment decision of any proposed offering [60] both at an individual consumer level but specifically at the organisational investor level.

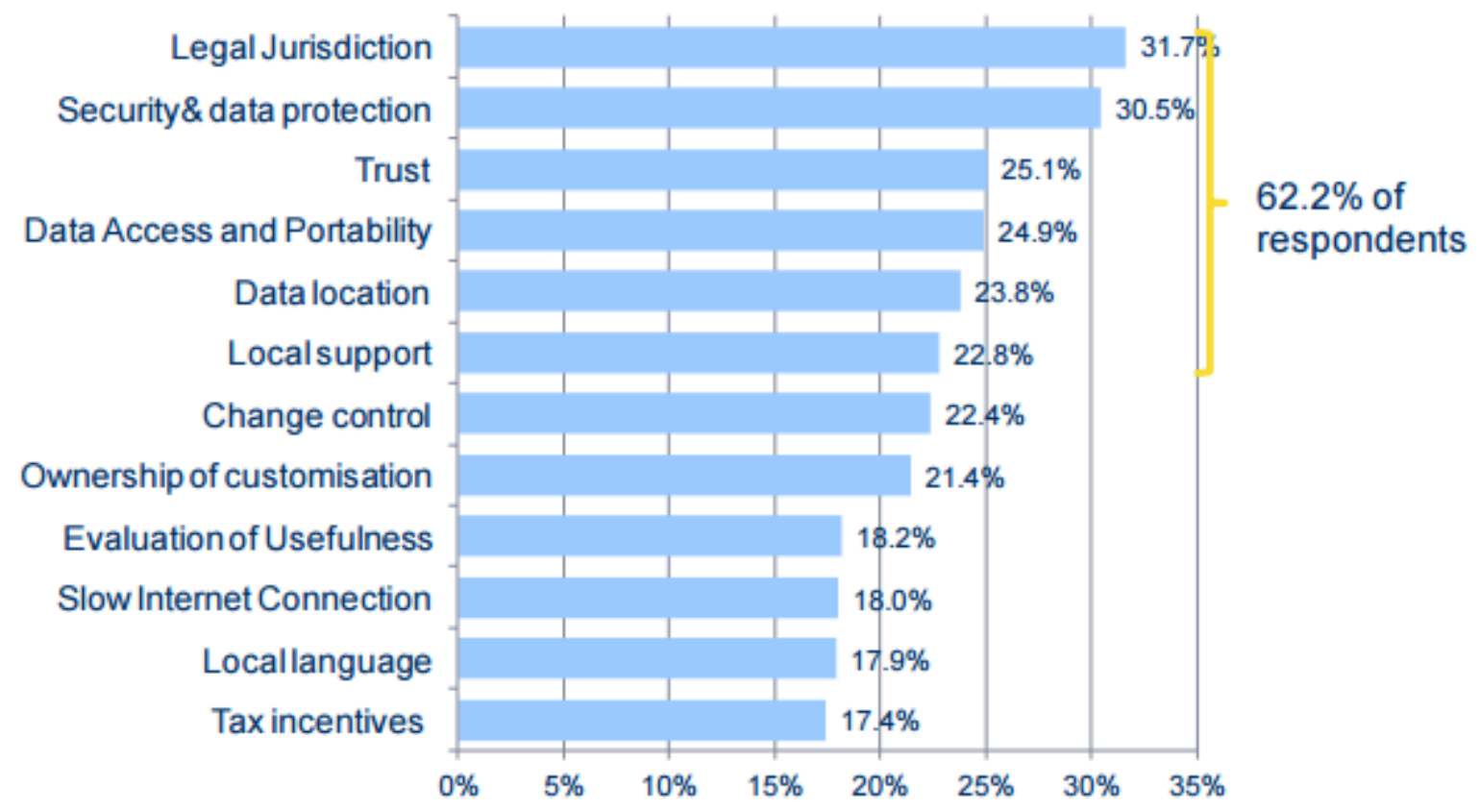

Figure 3: \% of respondents stating barrier is restricting (very/completely) cloud adoption [59]

\subsubsection{Technology standardisation}

An increasingly decentralised energy grid characterised by increased integration of DR and other Smart Grid resources, such as renewable energy sources, inherently results in increased complexity. Given the fact that nearly $90 \%$ of all power outages and disturbances have their roots in the distribution network [61], the risk from increased complexity from DR penetration is apparent.

Increased decentralisation and complexity drives the need for more complex information systems, to manage such complexity $[62,63]$. As described, DR increasingly relies on loT technology which leverages increasingly ubiquitous sensing (see section 3.1.3.1), both cloud and embedded computation (see section 3.1.3.2) and communications (see section 3.1.3.3). But there is apprehension in moving to what is perceived as uncertain technological trajectories. Standardisation is therefore key to adoption.

However, uncertainty regarding which standards will ultimately prevail may be a barrier. As per Figure 4 [6469] there are multiple posited loT reference models and architectures. The myriad of posited loT architectures pose uncertainty especially when viewed from the domain perspective. Will centralised cloud based solutions offer required rates of response? Or acceptable levels of security and privacy? Would decentralised approaches offer holistic visibility or scalability? Is a hybrid approach the most appropriate option? As Figure 2 and Figure 4 illustrate it can be arduous for potential end-users (e.g., industrial, energy, transport, built environment, water, agriculture etc.) to navigate and select appropriate compute and communication solutions/protocols. This can be a significant barrier to adoption as confidence in the quality of standards for DR enabling infrastructure is crucial for successful DR. For example, if communication is not robust enough, or not perceived to be robust enough, to offer an adequate quality of service (e.g., response rate to DR service calls, especially explicit DR called on to ensure network and system security [6]) DR adoption may be slow. 
Similarly, confidence is required in the ability of loT components to communicate with each other, e.g., to send control signals or to submit bids and offers. This echoes the link between market failure and imperfect information, see section 3.1.1.1. Without the necessary standardisation here flexibility cannot be identified and exploited [3]. Lack of standardisation on the interoperability of various components may also be a concern if it is thought that various devices may interfere with each other [45]. Further, lack of standardisation may prove a barrier if there is concern on the part of investors that they may become "locked-in" to a particular supplier. This may result in constraints on future decisions which lead to sub-optimal outcomes.

IOT Architecture -FP7 IOT-A

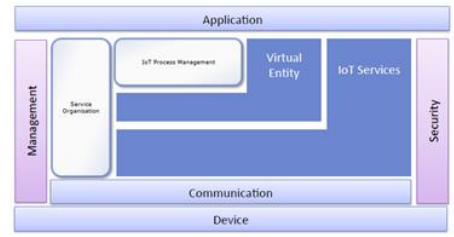

Intel IOT Reference Model

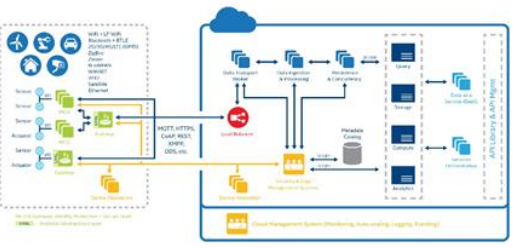

IOT World Forum Reference Model

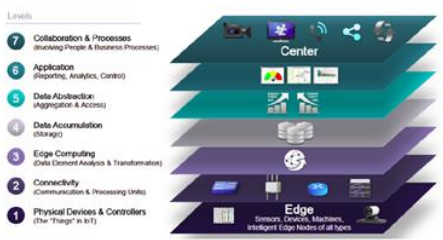

Open Internet Consortium

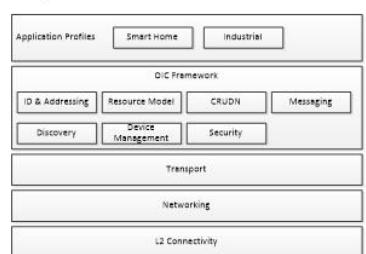

ITU-T Y.2060 IOT Reference Model

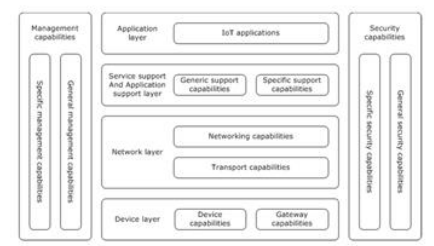

Industrial Internet Consortium

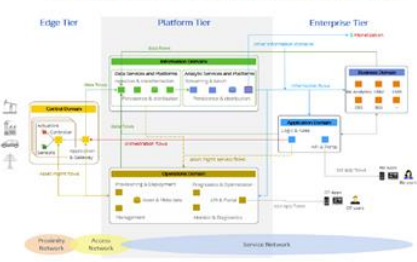

Figure 4: IoT architecture exemplars [64-69]

\subsubsection{Technological skills}

There is much that technology can deliver, but not at zero cost. User dissatisfaction and cost, can become significant barriers as a result of a poor requirement elicitation process, in which functionalities required by the user are not captured correctly and/or made available to technology developers. While not incorporating extensibility, flexibility, security, privacy by design is likely to cost more if attempting to apply retrospectively. Good design is a skill, requirement elicitation is a skill, having good local support as outlined in Figure 3 requires skilled resources. Which leads to another barrier, namely the recruitment and retention of technical talent.

IoT systems differ from the point-solutions of the past in their complexity and diversity of applications. This creates challenges in finding or training the right technical and domain expertise to design and build loT systems as well as to maintain, operate, and use them. Sometimes the problems stem from the uneven global distribution of technical talent. But often the issue is the mix of proficient skills required. By way of example Table 4 illustrates the skillset of the modern data scientist [70]. This issue, of acquiring a diverse but proficient skill mix, can be significant for the domain vertical (e.g. water, energy, transport) tasked with attracting such talent.

Table 4: Skills required of the modern data scientist [70]

\begin{tabular}{|c|c|}
\hline MATHS \& STATS & PROGRAMMING \& DATABASE \\
\hline $\begin{array}{ll}- & \text { Machine learning } \\
- & \text { Statistical learning } \\
- & \text { Experiment design } \\
- & \text { Bayesian inference } \\
- & \text { Supervised learning, decision trees, random } \\
& \text { forest, logistic regression } \\
- & \text { Unsupervised learning, clustering, dimensionality } \\
& \text { reduction }\end{array}$ & $\begin{array}{ll}\text { - } & \text { Computer science fundamentals } \\
\text { - } & \text { Scripting language e.g. python } \\
\text { - } & \text { Statistical computing package e.g. R } \\
\text { - } & \text { Databases SQL \& NOSQL } \\
\text { - } & \text { Relational Algebra } \\
\text { - } & \text { Parallel database and query processing } \\
\text { - } & \text { MapReduce concepts } \\
\text { - } & \text { Hadoop, Hive / Pig } \\
\text { - } & \text { Custom reducers } \\
\text { - } & \text { Experience with xaaS like AWS }\end{array}$ \\
\hline
\end{tabular}




\begin{tabular}{|c|c|}
\hline DOMAIN KNOWLEDGE \& SOFT SKILLS & COMMUNICATION \& VISUALISATION \\
\hline$-\quad$ Passionate about the business & - Able to engage with senior mgmt. \\
$-\quad$ Curious about data & - Story telling skills \\
$-\quad$ Influence without authority & - Translate data-driven insight into actions \\
$-\quad$ Hacker mind-set & $-\quad$ Visual art design \\
$-\quad$ Problem solver & $-\quad$ R Packages like ggplot or lattice \\
$-\quad$ Strategic, proactive, creative, innovative \& & $-\quad$ Knowledge of any of visualisation tools e.g. Flare, \\
$\quad$ collaborative & D3.js, Tableau \\
\hline
\end{tabular}

\subsection{Secondary barriers to DR}

Secondary barriers are those barriers relating to anthropogenic institutions, or system feedbacks, which result in some way to actions taken against the above fundamental barriers. These barriers generally relate to the power system, given its complex and regulated nature which is further increasing with the Smart Grid evolution. They can largely be classified as political/regulatory, as may relate to taxation, standards and regulation related to consumers, or monopoly activities, i.e., network operation. Further secondary barriers relate to market rules and the physical (network) constraints that DR can create.

\subsubsection{Political/regulatory}

Political/regulatory barriers are defined here as those barriers which exist as a result of government policies, usually enacted through regulation. In the literature the distortionary effects (on markets) of government policies are sometimes regarded as a market failure [28]. Such policies can result in barriers for a number of reasons. Firstly, markets can be distorted by the applicable tax code, which may treat various expenditures differently. Discrepancy in the treatment of operational/capital costs may cause a barrier when considering investment in DR-enabling technology, whilst tax discrepancy between substitutable goods (such as electricity and gas, or types of heaters) can cause distortion in the operation of DR. Another tax-related barrier can arise from the installation of electricity storage. When such storage lies behind a meter, tax will be charged on electricity used for charging the battery (as this cannot be separated from actual consumption). This will create a barrier to the efficient use of the storage [71].

Regulation may also cause distortion in markets if goods that are practicably substitutable (i.e., generation and consumption based operating reserve) are precluded from competing with each other. As highlighted in [71] this is part of a wider issue of the dominant paradigm in energy systems. Historically, electricity systems have built on the assumption that electricity flows from large scale central generators to passive, distributed users. Accordingly, the regulation of the system has been tailored to this paradigm. This can place barriers to DR, given the evolution towards the Smart Grid, when characteristics (such as minimum bid size, gate closure times and product definitions) are suitable for central generators, but not for demand side participants.

A general barrier to DR is regulation which prevents market price signals from reaching ultimate consumers. As detailed in [71] such regulation not only damages business cases for DR but also inhibits the efficiency of markets. Further, regulatory restrictions on locational/temporal price differentiation in markets will prevent consumers perceiving the true value of DR. In cases where transaction costs are thought to outweigh the benefits of full price-pass-through, or where net-metering, as an incentive for small-scale generation [71] is appropriate, it may be justified to retain regulation of consumer prices. Striking a balance between these motivations, so as to minimise overall barriers to DR is difficult.

A further political barrier may result from uncertainty derived from unclear policy ${ }^{1}$. Survey based literature has highlighted this as a particular barrier to smart grid development [72], which can be extended to DR.

Finally, given the heavily regulated nature of energy network operators, the barriers to DR posed by the regulation of network operators must be mentioned [73]. These include: the focus on historical performance,

\footnotetext{
${ }^{1}$ This may be considered a special case of the uncertainty market barrier, see section 3.1.1.2.
} 
rather than future requirements; short regulatory periods; focus on the network operator, rather than systemwide effects; and the lack of recognition of the value of research and development. In particular, the issue of different treatment of operational and capital cash flows is particularly relevant. Short regulatory periods, and the lack of uncertainty on the benefits of capital investment can encourage capital expenditure heavy grid expansion over operational expenditure heavy DR, leading to generally sub-optimal outcomes [10].

\subsubsection{Market structures}

Many DR business cases, which require action against a suitable 'baseline' profile, will require definition of a baseline methodology for the DR market to operate. Whilst high resolution metering data can describe the consumption/generation of the given resource, it cannot tell, in the event of a DR call, what the profile would have been without the call. To determine compliance with respect to the relevant contract, it is necessary that some baseline can be agreed between the buyer and seller. Indeed, as discussed in [17], establishing a baseline may be a barrier to deployment of DR, as it can impede proper valuation of a product.

Although energy and capacity (in which DR can operate) are continuous in nature, they are typically traded in defined products. These products are standardised according to a number of attributes, e.g., amount (kW or $\mathrm{kWh}$ ) or time of trade (week-ahead, day-ahead). If the definitions of the standardised products are too restrictive, they may preclude provision by some DR providers, or may mean that the full value of DR cannot be realised (resulting in sub-optimal system efficiency). Hence such standardisation may be considered a barrier to DR. On the other hand, it should be noted that standardisation of products generally reduces transaction costs, as the definition of a restricted number of products can reduce search and negotiation costs [36]. Thus, there may be tension between the motivations to reduce standardisation (to increase realised DR value and system efficiency), and increase standardisation (to reduce transaction costs).

A final, secondary market-related barrier may be the complexity of the resulting markets. Even with aggregation of DR resources [74], the number of DR agents may cause significant complexity in operation of Smart Grid/DR markets, especially if those DR agents wish to restrict the amount of information they reveal [43].

\subsubsection{Physical}

The increasing levels of resources and flexible loads responding to dynamic price signal may result in technical issues on distribution networks, where those resources/loads are connected to the electricity networks. This is since controllers will shift large portion of power consumption towards the least price periods, which in turn may overload distribution network assets and lead to voltage rise/drop issues, beyond the statutory voltage limits. This is demonstrated in Figure 5 which shows peak electricity demand, for a district of 100 flats, in the UK, on a winter weekday, with gas boiler heating, modelled using a version of the model presented in [56]. As shown the peak demand increases with the introduction of electricity storage and exposure to price signals, as the peak battery import ( $40 \mathrm{~kW}$ ) coincides with the start of the morning demand ramp-up around 7am. This is due to the electricity storage being used to shift district electricity consumption towards cheap times for use in middle of the day when prices are higher. Following the traditional planning approaches adopted by distribution network operators to maintain network constraints within limits, upgrading the distribution network assets is required. However, this may produce a technical barrier to DR, since network reinforcement is an expensive and time-consuming solution, resulting in a higher distribution network fees. 


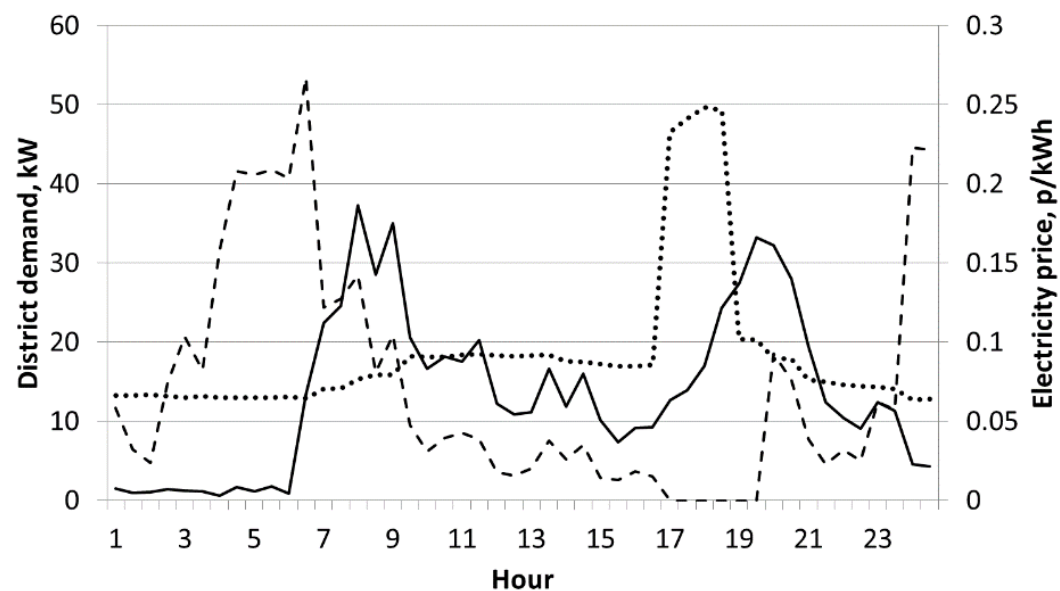

— No price signals. No battery storage - - Price signals, $4 \mathrm{kWh} / 0.4 \mathrm{~kW}$ battery

.... Electricity price

Figure 5: Net electricity demand for 100 houses, with and without battery storage and price signals

\subsubsection{Understanding}

As previously highlighted, a lack of understanding of DR, and the benefits that it may bring, is a considerable barrier to DR [7]. This barrier can be considered as connected to (indeed, a result of) other barriers, particularly many social and economic barriers (e.g., barriers related to imperfect information, access to capital, values etc.), whose existence are often not appreciated in existing assessment frameworks. Such a lack of understanding generally reduces interest in DR, which then results in less attention and investment from parties who may benefit from developing DR. In particular, a lack of understanding of DR restricts interest in development of appropriate tools, such as a systematic cost benefit analysis models for development of business cases [75]. In this way the lack of understanding of DR becomes self-perpetuating.

\section{Enablers}

Following the fundamental/secondary framework defined for classification of barriers to DR, enablers, to counter the defined barriers, are defined, below. The detailing of an enabler below does not necessarily constitute a recommendation for its adoption, as enablers typically have a cost associated with them. Indeed, any action to enable DR should be subject to a cost-benefit analysis, to ensure cost-effectiveness. Further, practitioners should be cognisant of the possibility for unintended consequences (feedbacks) from enabling actions, which may hamper (or, indeed, enhance) DR adoption.

\subsection{Fundamental enablers of DR}

\subsubsection{Enablers against economic barriers}

Economic enablers will either relate to improvement in the functioning of Smart Grid markets through fixing market failures or intervention in market operation, specifically to address some market barrier.

\subsubsection{Enablers against market failures}

An initial enabler of DR can be improved understanding of the potential DR resource. Studies have shown that simple measures to increase understanding can have significant impact on DR [76]. A step towards improving the functioning of markets, with particular regard to DR, can be the development of markets specifically for trading of demand-side flexibility, or for adjustment of existing markets $[2,77]$. Such enablers could reduce informational barriers by reducing search costs which may be a significant proportion of potential profit for DR providers, by bringing together buyers and sellers, as demonstrated in Figure 6 . 
a)

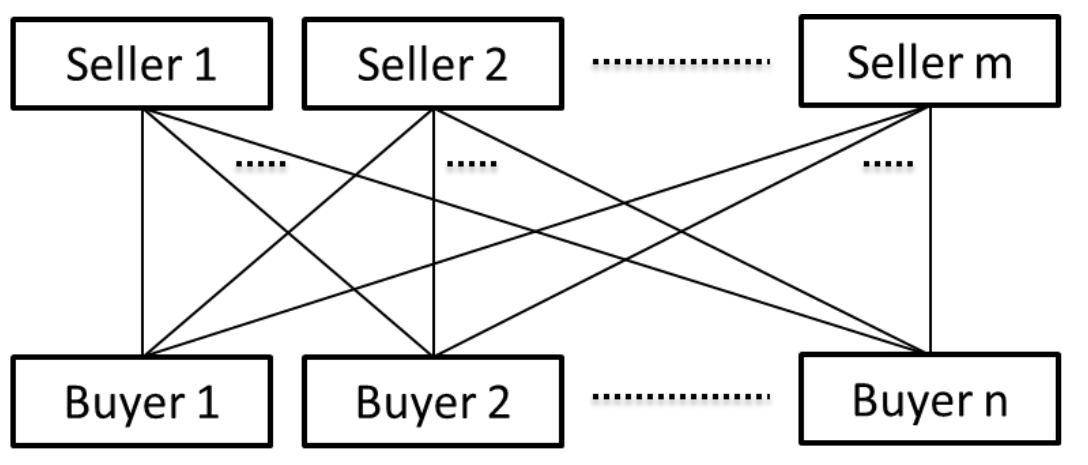

b)

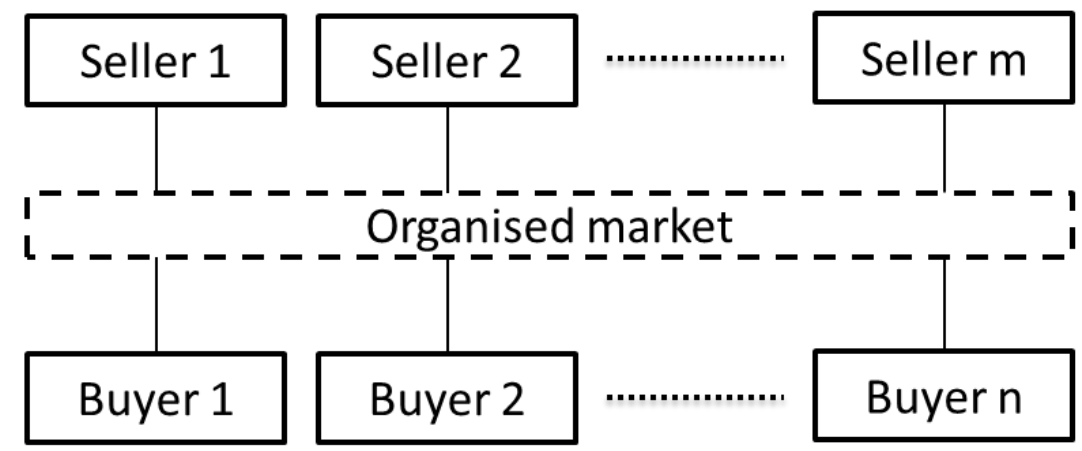

$\mathrm{m} \times \mathrm{n}$

connections

$\mathrm{m}+\mathrm{n}$

connections

Figure 6: Number of connections for buyers/sellers to search a) without an organised market, and b) with an organised market

Enabling DR, through addressing the issue of 'split incentives', is particularly difficult. This is because user preferences (which the DR aggregator agent should take into account when acting on behalf of the DR provider) are ill-defined, probably time variant, and probably not understood fully by the users themselves [78]. Here efforts should focus on development of metrics of user preferences that might enable quantification and trading of flexibility (such as metrics on thermal comfort [56]). Such metrics may then reduce the impact of split incentives by producing a means to effectively communicate information on user preferences. $A$ further enabler of DR may be development on the design of contracts, to better signal efficient behaviour to DR providers, thus increasing the available benefits. Reference [34] highlights the necessity of contracts to capture the preferences of consumers, which may require a wide variety of contracts.

To address the problem of incomplete markets (see section 3.1.1.1), there is clearly a requirement to account for externalities, such as $\mathrm{CO}_{2}$ emissions, ideally through market-based emissions trading schemes. The issue of 'free riding', as multiple parties benefit from the exercising of DR may be solved through concepts such as a 'DR exchange' [31], which can assign the costs of DR according to the received benefits. Similar solutions are being explored by industry parties $[2,79]$.

To deal with barriers of imperfect competition, regulators must be able to monitor market power in DR markets. This may especially be an issue in local markets, in which there are few participants, such as for distribution network constraint management, or similar [80]. Countering market power may also be aided by development of the aggregator concept [14], or platforms enabling peer-to-peer communication [81], and thus coordination and optimisation, see section 4.2.2. This would enable access of DR to various markets, increasing the efficiency of those markets.

\subsubsection{Enablers against market barriers}

The above detailed enablers relate to action to improve the functioning of markets. To address market barriers, it is necessary to intervene in markets to deal with features which are natural results of the market. 
Therefore, enablers to reduce barriers related to access to capital, uncertainty, hidden costs or value generally, require market intervention. However, if the enablers result in social benefit, as exploitation of DR will, then such market intervention can be justified by governments.

Specifically, DR may be enabled by subsidy of various types. Loans may be offered at reduced rates, or guaranteed by government, to reduce barriers related to access to capital (such as with the UKs Green Deal [82]). If revenues are uncertain, tools such as contracts for difference can be offered to ensure a minimum payback [83]. Similarly, hidden costs, such as transaction costs, may be dealt with by subsidy of a market which offers to handle negotiation and enforcement of contracts. It may be argued that DR can be enabled by similar intervention to deal with low system value for flexibility. However, if there is an inherently low system value for flexibility in a system, it is unlikely that any government will want to subsidise the participation of flexible parties, such as DR providers, in energy markets. As previously mentioned though, the system value of flexibility can change over time. Given this uncertainty, assessment methods which enable assessment under long term uncertainty, such as real-options-based methods may be considered an enabler of DR [84,85].

\subsubsection{Enablers against social barriers}

Given the objectives of organisations to be profit-making, proliferation of information showing the cash benefit of DR should be a significant enabler, to counter barriers of (lack of) power for the relevant decision maker, and culture within an organisation. However, as well documented, it may not be as simple as that as organisations do not always act perfectly rationally [86]. Nevertheless, increase in status for DR decision makers should help to enable DR. To counter any cultural barrier, a general education on the benefits of DR should be implemented.

Enabling individuals to change their behaviour to promote DR participation may be more difficult. Where behaviour to promote flexibility is constrained by bounded rationality (i.e., cognitive limits on the processing of information, which may relate to the time available, also), a significant enabler may be automation [87]. Automation, such as 'Energy boxes' [3], smart thermostats, or building energy management systems, may enable DR by making operational decisions which the user is unable or unwilling to. This may also reduce inconvenience for the user (as they has effectively delegated decision making), further enabling DR. However, as such technology is unlikely to be able to fully capture user preferences, an important feature is the requirement for an 'opt-out' function [88]. This, however, will inevitably affect the value of DR, though this can be mitigated through adequate portfolios of resources. The likelihood of opt-out, and hence the likely degradation of DR value, can be mitigated through appropriate selection of DR business cases. For example, if DR providers have low tolerance for lost comfort, capacity-related DR (i.e., network congestion management) may be more appropriate than energy-related DR (i.e., energy arbitrage) [10]. Such technology may also be important in countering barriers of information presentation. Intuitive and clear information will enable DR by ensuring information is perceived as intended by the sender [89].

This leads on to discussion on user preferences, which derive from user values. The process of influencing user preferences to enable DR (e.g., attitudes to trading convenience or comfort for cash, or to allowing third party control of devices) is much less straightforward. As described in [40] the evolution of preferences is very complex, involving multiple feedbacks and, particularly, co-evolution with relevant institutions. There is no quick enabler here, though user values may be influenced by efforts to change institutions (e.g., changing social norms by influencing perceptions of energy use, or changing laws and regulation to communicate those changed norms). A related point is how to deal with the inertia barrier. Again, there is no quick fix, though ensuring information is transmitted correctly can ameliorate inertia barriers, by making sure users are fully aware of benefits. Further, gradual change (e.g., from flat rates, to two-rate time-of-use tariffs, then to dynamic real-time tariffs) should be employed, to ensure inertial forces do not result in rejection of DR-friendly behaviour [16]. 
Enabling DR through addressing concerns of trust may be more practical. Whilst trust in existing energy system actors may be low [3], DR may be enabled through partnering with new third-parties that may proliferate in the Smart Grid (such as aggregators), circumventing issues of low levels of trust with existing actors. Indeed the source of information has been shown to key to motivating DR [90]. Increased trust between DR providers and aggregators may also assuage concerns on privacy, as end-users are more likely to be happy to share information (such as meter profiles) with parties who they trust. Whatever the levels of trust, a principle that should be central to all DR activities should be the ownership of consumer data by the consumer [3]. If this principle is clear, possibly enforced legally, consumers may have more confidence in DR. Concerns on privacy may also be addressed through technological solutions. End-users may be given confidence that their data cannot be exploited to obtain personal information, through data anonymization [91]. Further, a general principle of modular/cellular design can increase security, or rather reduce the impact of a successful attack, whilst giving users the ability of users to tag their data, to manage the life cycle of that data, may be an enabler, by building the confidence of users. Further, enabling user choice on where data is archived (on the premises or in the cloud) may be an enabler, by placing decisions on data storage in the user's hands.

It should be noted, however, that such user acceptance barriers may decline in relevance over time, as institutions, e.g., 'hard' institutions, such as markets and laws, and 'soft' institutions, such as social norms, change over time [40].

\subsubsection{Enablers against technological barriers}

In a Smart Grid context, technological enablers to DR will relate to improvement of sensing, computation and communication/actuation, within the ICT realm, to standardisation or to more social ICT-related elements, related to technological skills.

\subsubsection{Enablers against sensing barriers}

A clear enabler with respect to DR is the installation of metering at the required resolution. This is currently underway in many developed electricity systems [92,93]. However, further development may be needed on this front if DR resources wish to partake in "fast" reserve markets, such as frequency regulation, which may require higher resolution metering. If storage within a property is to be metered separately (see section 4.2.1), this may also require more investment.

To counter barriers to DR stemming from inadequate monitoring of final energy services (e.g., thermal comfort), relevant management systems should incorporate monitoring, not only of electricity consumption, but also of final energy services. This should be informed by user preferences, which are likely to vary, see section 3.1.2.2. The challenges of specifying such systems illustrate the importance of requirement elicitation, relevant in the development of all DR-related technology. To ensure alignment between technology design/development and required application and deployment, the best known requirement engineering practises should be adopted. Good requirement elicitation practises will assist in ensuring correct identification of the desired service/use case, thus reducing technology misalignment and/or misuse. To this end, user led design, use-case development, and ethnography should be employed for all Smart Grid/loT products/services. The importance of truly understanding what a customer/user is trying to achieve in purchasing and/or commissioning technology cannot be underestimated, but is often poorly done.

Incorporating user design principles and using ethnographic expertise in understanding the use-cases can save considerable frustration and cost, further into the development and operation cycle. To aid clarity on technology capability, succinct, clear documentation, specifications and user guides should be ensured. This removes uncertainty regarding technology fit, and ensures the technology is used as designed, given the usecase.

Device abstraction layer technologies (i.e., technologies that act as translators for multiple sensor protocols), are outlined in compute enablers (see section 4.1.3.2) but could just as easily be discussed here as sensors, sensor data acquisition and compute are all interweaved in enabling multi-protocol sensors/actuator services, 
required for DR. Section 4.1.3.4 deals more specifically with standards while some more sensor specific exemplars targeting semantics follow.

SensorML (Model Language) [94] - The primary focus of the Sensor Model Language (SensorML), developed by the Open Geospatial Consortium (OGC), is to provide a robust and semantically-tied means of defining processes and processing components associated with the measurement and postmeasurement transformation of observations. This includes sensors and actuators as well as computational processes applied pre and post measurement. The main objective is to enable interoperability, first at the syntactic (i.e., format) level and later at the semantic (i.e., meaning) level.

SensorSSN (Semantic Sensor Network) [95] -The SSN ontology describes sensors and observations, and related concepts. It does not describe domain concepts such as time, locations, etc. These are intended to be included from other ontologies via OWL (web ontology language) imports. The ontology was developed by the W3C (World Wide Web Consortium) Semantic Sensor Networks Incubator Group (SSN-XG), again, in order to aid interoperability.

Semantic Sensor Web [96] -SSW suggests an approach to annotating sensor data with spatial, temporal, and thematic semantic metadata. This builds on the aforementioned standardization efforts within the W3C and OGC and extends them with semantic Web technologies to provide enhanced descriptions and access to sensor data.

\subsubsection{Enablers against computing barriers}

The complexity generated by the large amounts of data, and the many sources of uncertainty, may be ameliorated by the simplification of any optimisation. Time-steps may be lengthened, resources may be aggregated, and the number of scenarios (in a stochastic optimisation) may be limited. The question, which may often not be definitively answered, is: what is the appropriate trade-off between reduced complexity, and increased accuracy? The answer may change as computational power driven by 'Moore's Law' continues to become cheaper, more efficient and more powerful [97], particularly in the embedded space.

In particular, development of big data technologies, when used in combination with domain heuristics can deliver real insights in coping with complexity. Machine-learning techniques are specifically useful in that regard. More generally, adoption of a system-of-systems approach $[98,99]$ is one means of coping with complexity, by distributing computational burden around individual systems, while providing recommender functionality at the aggregated level. Such an approach may be aided through scalable cloud computing solutions, though this then may be limited by communication barriers specifically related to trust and interoperability.

In the shorter term DR may be enabled through adoption of appropriate multi-protocol gateways, which abstract and link physical 'things', compute devices, management systems and the internet. Such gateways may adopt open device abstraction technologies such as OpenHAB [100], loTivity [101]. In a world of multiple wireless protocols, such technologies act as one-to-many translators reducing complexity and allowing for common communication. The Open Interconnect Consortium (OIC) is an example of standardisation efforts in this area [68].

In a similar vein, development of plugin-based architectures might prove valuable. Plugin-based architectures promote modularity and extensibility, allowing parallel development, aiding new feature/adapter creation. This should promote cross-sector and third-party development, and hence adoption. Allowing for composability is seen as a key enabler in addressing interoperability and is a specific aspect discussed within the Industrial Internet Consortium (IIC) architectural documentation [69]. 


\subsubsection{Enablers against communication barriers}

As per section 3.1.3.3 interoperability (or lack of it) can be a major barrier to communications at scale, if the heterogeneous protocols of existing systems cannot be bridged to allow for shared unambiguous meaning, then the transactions required to deliver DR at scale cannot be communicated and DR has no chance of being delivered at scale. However, while Figure 2 outlines the myriad of existing standards and alliances which need to be navigated, it also highlights the efforts being made to deliver interoperable information exchange in the Smart Grid. Standardisation in terms of data formats, transfer mechanism and semantics are all being progressed and will undoubtedly contribute to defining communication standards required at the loT scale.

Partnership and the formation of alliances promoting cross-sectorial collaboration is one valuable means of driving standardisation, see section 4.1.3.4. Regulation may be required to hone focus around key standards. Initiatives such as LinkedData and OpenData may also be powerful means of enabling DR [102,103]. Overall, greater priority should be on semantics, as establishing a common understanding is likely to prove more difficult than delivering technical standardisation, as outlined in sections 4.1.3.1 and 4.1.3.4.

As discussed, security regarding information exchange (see section 3.1.3.3) is a significant barrier to adoption and hence energy services. Table 5 outlines security aspects that need to be considered in any holistic approach to security.

If such elements are incorporated at the development stage (i.e., security by design), this can considerably mitigate concerns. Privacy by design should also be considered. Incorporating privacy aspects at the data model level should help with appropriate data lifecycle management. Additionally, developing interfaces and mechanism for allowing users to tag data in intuitive ways could offer a means of mitigating uncertainty regarding privacy legislation which can be a barrier to investment in loT infrastructure.

Table 5: DR security considerations

\begin{tabular}{|c|c|}
\hline Network aspects & Other aspects \\
\hline $\begin{array}{ll}\text { - } & \text { Firewall } \\
\text { - } & \text { Virtual private networks } \\
\text { - } & \text { Authentication }\end{array}$ & \multirow{3}{*}{$\begin{array}{ll}\text { - } & \text { Key management } \\
\text { - } & \text { Device attestation } \\
\text { - } & \text { Runtime controls } \\
\text { - } & \text { Stack simplification } \\
\text { - } & \text { Integrity measurement } \\
\text { - } & \text { Data encryption } \\
\text { - } & \text { Data authentication }\end{array}$} \\
\hline Physical aspects & \\
\hline $\begin{array}{ll}\text { - } & \text { Device-specific cert } \\
\text { - } & \text { Trusted Platform Module Platform } \\
\text { Configuration Registers } \\
\text { - } \quad \text { Secure boot } \\
\text { - } \quad \text { Physical access }\end{array}$ & \\
\hline
\end{tabular}

Additionally, improvements in the reliability of wireless sensor networks [104] are required to enable trusted applications, DR included. Sub $1 \mathrm{GHz}$ protocols and mesh based approaches offer potential compared with more established, but increasingly congested protocols in the $2.4 \mathrm{GHz}$ range [105].

\subsubsection{Enablers against technology standardisation barriers}

As has been highlighted in Figure 2, efforts are being made to deliver interoperable information exchange. However, further consolidation and convergence of standardisation is required as is the development of a shared taxonomy between the energy, built environment and ICT sector.

Some moves have been made in this direction. For example, the energy industry is adopting different ICT terminologies for the partitioning of the command-and-control layers of the Smart Grid (e.g., home, local and wide area networks) [61]. The eeBDM (energy efficiency building data models) community has looked to 
collate and consolidate various energy and building related taxonomies, ontologies and data models [106]. The European Commission launched a study on available semantics assets for the interoperability of smart appliances, promoting a common ontology as a M2M (Machine-to-Machine) application semantics layer, SMART 2013/0077 [107]. The EC hoped this will provide background material enabling all stakeholders to discuss a single ontology for home appliances. The European telecommunications standards institute (ETSI) seconded this approach by creating the Semantics For Smart EE project, producing several interim study reports $[107,108]$.

Additionally, building information model standards (e.g., the Industrial Foundation Class and the Information Delivery Manual [109]) are extending energy efficiency aspects pertaining to smart grid interaction. Meanwhile some IoT/ICT focused alliances are increasingly looking to consolidation e.g. the acquisition of the assets of the UPnP (universal plug and play) Forum by OIC, the strategic alliance between OIC and IIC, OpenHAB with the Eclipse SmartHome project etc. Such efforts if accelerated could significantly mitigate barriers to DR services by reducing the complexity of choice with respect to data exchange standards.

\subsubsection{Enablers against technological skills barriers}

As outlined in section 3.1.3.5 the recruiting and retention of loT talent to support domain services can be an arduous process. Part of the issue is knowing exactly what type of skills one requires for any given scenario. Additionally, acquiring talent with a tolerance of ambiguity and a propensity for continual learning is a must, given the inherent uncertainty of the fast changing tech sector. Knowing what skills are needed and how strategically important they are to one's business context is essential. If not strategically important, or if uncertainty is very high, outsourcing could be considered as an alternative, as this offers the flexibility to trial and hone skillset requirements and to modularly adjust more rapidly in the short term.

Once the decision is made to hire, one needs to consider how to attract and keep the right talent. A survey of industry professionals has indicated that the top three incentives for staying in their current jobs, their answers were: more money, better work/life balance and a flexible work schedule or telecommuting. Also highly cited, opportunities for professional development, especially if they resulted in advancement [110]. Experts suggest eight things to consider in attracting and retaining technical talent [111]:

1. Include in decision making

2. Don't micromanage

3. Flexible work hours

4. Invest in training

5. Provide access to new tech

6. Give praise $\&$ acknowledge contributions

7. Offer free stuff

8. Provide a competitive compensation package

\subsection{Secondary enablers of DR}

\subsubsection{Enablers against political/regulatory barriers}

To counter the barrier caused by tax being charged on energy used for charging storage, separate metering of different types of resources may be implemented. This disaggregation of consumption, generation and storage can result in more efficient taxation, e.g., through avoidance of taxing charging of storage. A wider enabler is the more general review of energy system regulation to identify regulation which is a product of the legacy dominance of central electricity generation, and which unnecessarily inhibits Smart Grid solutions, such as DR. 
This is clearly a significant task, and requires the support of all stakeholders, some of whom may have entrenched views of the substitutability of demand-side resources for generation resources in many areas.

A more specific enabler would be the ability to pass price signals, possibly through a third party, to end-users, to promote efficiency in energy markets. Whilst the demand amongst end-users for such capability (given the transfer of market risk to consumers that this would entail) is not certain and is likely to vary, regulators should offer the option. As with any new service/technology, adoption of the option may take time, but the growth of enabling technologies (e.g., smart meters, home automation, broadband internet) means that option may be increasingly attractive.

Further, cost-reflectivity in energy markets should be improved generally, to improve market efficiency. For example, penalties for imbalance which do not fully reflect the costs of the imbalance (e.g., due to reserve provision and balancing mechanism operation) is a regulatory factor which inhibits efficient market operation. Measures should be taken, where cost effective, to make sure all markets are cost reflective. An example can be the measures being taken in the UK to reform imbalance pricing in the UK [112].

With regard to grid operator regulation, greater emphasis on innovation and new solutions (such as distribution network constraint management), such as is encouraged by the 'revenue+incentives+innovation +outputs' (RIIO) framework in the UK [113]. In general, policy certainty is an enabler, to counter perceived uncertainty, which may inhibit commitment to DR. 'Forward guidance', signalling the conditions under which policy may change may provide some certainty, though it will reduce flexibility for policy-makers.

\subsubsection{Enablers against market structures barriers}

To enable DR to be traded in (dedicated or general) markets, agreed baseline methodologies are required. Such baseline methodologies, which are acceptable to all parties, will enable markets which rely on "explicit" DR (trading load) [6] to operate. Several types of baselines are possible (such as historic, maximum base load, meter before/meter after), as discussed in [114]. With respect to standardisation in markets, to better realise the potential of $D R$, it may be necessary to review the standard definition of products. However, there is no clear policy here, as, in all cases, the benefits from any relaxation in standards must be weighed against the associated rise in transaction costs. Standardisation of products in markets is linked to the wider issue of complexity, which will increase as the number of options, in various areas of the electricity system, increase.

With regard to the barrier of complexity, there is uncertainty on how to enable DR. Complexity may be ameliorated by avoidance, if possible, of complex commercial arrangements, which entail significant communication between distributed parties. Examples include decentralised optimisation approaches, e.g., Lagrangian relaxation based methods [115], or game theory based local markets [116]. There is, however, some tension here, as distributed, 'systems-of-systems' approaches are likely to be significant features of the Smart Grid, and be an enabler of DR, through the limitation of computational complexity, see section 4.1.3.2.

\subsubsection{Enablers against physical barriers}

An alternative to network reinforcement, which can enable DR, is the management of distribution networks in real time, using DR. To do so, adequate real-time pricing mechanism should be in place to encourage DR providers to change their power scheduling so that network constraints can be managed effectively. A decision-making algorithm is needed to determine the required maximum power consumption/injection from premises to distribution networks at the time of network issue. The corresponding maximum power cap depends on the premises location in the distribution networks [117]. To further encourage participation in the management of distribution network constraints, a percentage of the reduction in distribution network investments can be used as a DR incentive. 


\subsubsection{Enablers against lack of understanding}

To counter barriers relating to a lack of understanding of DR, suitable cost benefit analysis frameworks must be developed. Such a framework must be able to accurately represent the decision making of the DR provider, which may be far from rational, in the classical economic sense [40]. They must also accurately appreciate all the possible markets for DR, and the power of new commercial arrangements, such as aggregation. Although some progress has been made in this direction, there is much farther to go [74].

\section{Discussion}

Drawing on previous sections Table 6 summarises the barriers and enablers of DR. As demonstrated, the barriers to DR are numerous. A fact that is, perhaps, belied by the division of barriers and enablers into classes (necessary for clarity in this complex area), is the connections between different barriers, enablers, and, indeed the wider electricity and energy systems in which DR exists. This may be relatively clear for secondary barriers, but perhaps less so for fundamental barriers. For example, the secondary barrier of network constraints results from a system feedback, as end-users respond to system behaviour (i.e., reduced electricity price at certain times), shifting demand to increase their utility. For the more fundamental barriers, however, there is also a high degree of connectedness. For example, enablement of DR through improved automation (aided by convergence of standards) may reduce DR, as preferences change, and customers become less engaged [41]. Similarly, barriers to DR may arise, through regulation, if adoption of DR reduces equity amongst energy consumers, offending preferences for 'fairness' [41,118]. Effects within the DR system may, also, be less straightforwardly causal. For example, there is likely to be complex co-evolution of preferences and institutions in relation to the Smart Grid. This relates to 'hard' institutions such as markets and regulations, but also 'soft' institutions such as social norms with respect to social acceptability of DR [40]. Indeed, these features of feedback, emergence and co-evolution suggest that the social, economic, and physical, energyrelated, system in which DR exists is a classic example of a complex system [119]. An implication is that further analysis of DR, and of other energy system (especially demand side) interventions need to acknowledge the complexity of the system, possibly through application of approaches from the emerging field of complexity science [120]. In particular, incorporation of the 'human dimension' has been highlighted as necessary to understand energy systems as a whole [121]. This has been identified as a priority by the UK energy regulator [122], to mitigate resultant uncertainty and risk, which can result in undesirably large risk premiums and delays to investment $[123,124]$.

Table 6: Summary of barriers and enablers of DR

Barriers

Economic

Market failures

Imperfect information

$\overline{\widetilde{\pi}}$

markets

Imperfect competition

Market barriers

Access to capital

Uncertainty

Hidden costs

System Value
Enablers

- Development of bespoke DR markets to bring together buyers and sellers, improving access to information.

- Develop metrics for effective communication of user preferences, to enable quantification and trading of flexibility.

- Pricing of externalities, such as emissions.

- Adoption of 'DR exchanges' or similar, to eliminate 'free riding' in DR exercise.

- Monitoring of market power, especially in markets with few participants.

- Preferential (government backed) loans.

- Contracts for difference.

- Subsidy of DR market operating costs.

- Long term evaluation methods (to appreciate the possible changing value of DR). 
Social

Organisational



Culture

- General education on DR and its benefits.

Behavioural

Form of information $\quad$ • Careful design of user interfaces.

Credibility and trust $\quad$ - Penetration of new, third-parties (such as aggregators).

- Legal clarity on data rights.

- Modular design of IT systems, to increase security.

- Data anonymization.

Values $\quad \bullet$ Evolution of DR institutions

\begin{tabular}{l|l}
\hline Inertia & $\bullet$ N/A \\
\hline Bounded rationality & $\bullet$ Automation.
\end{tabular}

Technological

Sensing

\begin{tabular}{l|l} 
Metering & $\bullet$ Installation of metering at necessary resolution.
\end{tabular}

Energy service sensors $\quad$ • Monitoring of final energy services (e.g., comfort, appliance availability etc.).

- Good requirement elicitation, to ensure DR schemes compensate according to user preferences on various energy services.

Computing

Computing power

- Optimisation simplification.

- Distribution of computation load.

- Leveraging of additional network resources, e.g., cloud

Communication

Interoperability

- Open, agnostic technologies.

- Plugin-based architectures.

- Alliances/collaboration to develop standards.

- Agreement on semantics, to develop a common language across industries.

Data security \& Privacy

- Adopt Security \& Privacy by design

- Have a data life cycle mgmt. strategy

- Allow for intuitive end-user configuration tagging of data

- Adopt current state of the art

Technology standardisation

Multiple competing

standards

Technology skills

Insufficiently skilled

workforce
- Consolidation of standards amongst energy, building and ICT sector alliances.

- Outsourcing as an option.

- Address identified factors for retaining talent.

- Know the requisite skills mix

- Target continual learners with tolerance of ambiguity

Political/regulatory

Taxes

Energy markets

regulation

End-user price regulation

Unclear policy

Network operator

regulation application of taxes.

- Systemic acceptance of the equivalence of demand and generation resources in energy markets.

- Improve cost reflectivity in energy markets.

- Allow end-users to face full variation of wholesale energy-related markets.

- Forward guidance on energy policy.

- Greater emphasis on innovation and new solutions.
- Disaggregation of consumption, generation and storage, to enable 


\begin{tabular}{|c|c|}
\hline \multicolumn{2}{|l|}{ Market structures } \\
\hline Baselines & - Agreed baseline methodologies, for various markets. \\
\hline Product definition & $\begin{array}{l}\text { - Review standard definition of products in energy markets (bearing in mind } \\
\text { the effect on transaction costs). }\end{array}$ \\
\hline Complexity & - Decentralised optimisation (system-of-systems approach). \\
\hline \multicolumn{2}{|l|}{ Physical } \\
\hline $\begin{array}{l}\text { Distribution network } \\
\text { constraints }\end{array}$ & - Real-time network pricing. \\
\hline \multicolumn{2}{|l|}{ Understanding } \\
\hline $\begin{array}{l}\text { Lack of understanding of } \\
\text { DR }\end{array}$ & $\begin{array}{l}\text { - Cost-benefit analysis framework, to demonstrate benefits of DR, under } \\
\text { realistic assumptions. }\end{array}$ \\
\hline
\end{tabular}

Given the demonstrated significance of social barriers to DR, another implication is that DR barriers may be lessened if the individual can be removed from its exercise. A systemic way to achieve this, without degradation of control over final energy services and resultant comfort/convenience, is through implementation of energy-service based solutions [125]. In this way the energy service provider will take decisions on DR provision, whilst being contractually bound to deliver energy standards to agreed standards. This can be beneficial to promotion of DR as such entities (as firms) are likely to be less prone to behavioural biases, and be less affected by market barriers, e.g., related to uncertainty, and access to capital, due to their larger size and specialised competencies.

As acknowledged by other authors a result of the demonstrated complexity around DR is the lack of appreciation of DR and its potential profitability, which is likely to increase [126]. This can itself, be conceived of as a barrier to DR $[7,17]$. Given the social benefit from the increased energy system flexibility DR would bring (probably at low cost compared to the alternatives: flexible generation, storage and interconnection [1]), there may be justification for political intervention to increase awareness of DR, and the challenges to its implementation.

Lastly, returning to the idea of co-evolution, it should be highlighted that DR cannot be considered separately to developments in the wider energy system, such as development of the Smart Grid. In particular, although DR enjoys some inherent advantages over other types of electricity system flexibility (e.g., DR is ideally placed to replace or defer distribution network enhancement, without investment in dedicated infrastructure, such as storage $[10,127])$, other flexible technologies may become dominant. This may be a likely scenario, as the barriers to their implementation are largely technological, and technological barriers are usually more tractable than the complex, uncertain social barriers which are relevant for DR. Notwithstanding this, it is possible that DR barriers may be overcome more easily, if necessary 'tipping points', perhaps resulting from self-catalysis amongst sets of DR provider preferences and relevant institutions, are reached $[119,128]$. Clearly suitable approaches for dealing with these social barriers (drawing on the enablers described in this work) are required to facilitate $D R$.

\section{Conclusion}

In this work barriers and enablers for DR have been classified and explored, in a Smart Grid context. A summary is given in Table 6 . Barriers have been initially classified as fundamental and secondary. Fundamental barriers include those classes of barriers which relate to intrinsic human nature (social/economic barriers), and to essential enabling technology (technological barriers). Secondary barriers relate to anthropogenic institutions (regulations, markets), or behaviour that results from feedbacks in response to DR participation (physical constraints). These classifications cover DR barriers (and also enablers) from the two key perspectives of the Smart Grid, namely, those of the power system and ICT (the "internet of things") industries. Based on the classifications of barriers, possible enablers of DR are explored. A strength of this work is the clarity it brings regarding barriers and enablers of DR, by producing a comprehensive and discrete classification. 
However this classification does belie the complexity of DR and energy systems generally. To acknowledge this complexity the inter-relationship of different barriers is explored and the relevance of the existence of signature characteristics of complex systems (e.g., co-evolution, emergence, feedbacks) is discussed. By defining the fundamental barriers and enablers of DR it is hoped that this work may act as a reference for further studies on development and modelling of DR, and as a bride to further study on the complexity of DRrelated systems.

\section{Acknowledgements}

Many thanks to Eduardo (Alex) Martínez Ceseña, Sereen Althaher and Nick Chapman for interesting general discussions, which were useful for clarifying and developing ideas. Further, the authors are grateful for the inputs provided by all partners in the COOPERaTE and DIMMER projects, and to the European Commission for the economic support under grants 600063 (COOPERaTE) and 609084 (DIMMER).

\section{References}

[1] Strbac G, Pudjianto D, Djapic P, Gammons S. Understanding the Balancing Challenge. 2012.

[2] EURELECTRIC. Flexibility and Aggregation Requirements for their interaction in the market. 2014.

[3] Losi A, Mancarella P, Vicino A. Integration of demand response into the electricity chain: challenges, opportunities, and Smart Grid solutions. London, UK; Hoboken, New Jersey, USA: Wiley-ISTE; 2015.

[4] Siano P. Demand response and smart grids-A survey. Renew Sustain Energy Rev 2014;30:461-78. doi:10.1016/j.rser.2013.10.022.

[5] Stoll P, Brandt N, Nordström L. Including dynamic CO2 intensity with demand response. Energy Policy 2014;65:490-500. doi:10.1016/j.enpol.2013.10.044.

[6] SEDC. Demand Response : Clarification of the standard processes required between BRPs and independent aggregators. 2015.

[7] Strbac G. Demand side management: Benefits and challenges. Energy Policy 2008;36:4419-26. doi:10.1016/j.enpol.2008.09.030.

[8] O'Connell N, Pinson P, Madsen H, O'Malley M. Benefits and challenges of electrical demand response: A critical review. Renew Sustain Energy Rev 2014;39:686-99. doi:10.1016/j.rser.2014.07.098.

[9] Drysdale B, Wu J, Jenkins N. Flexible Demand in the GB Domestic Electricity Sector in 2030. Appl Energy 2015;139:281-90. doi:10.1016/j.apenergy.2014.11.013.

[10] Martínez-Ceseña EA, Good N, Mancarella P. Electrical network capacity support from demand side response: Techno-economic assessment of potential business cases for small commercial and residential end-users. Energy Policy 2015;82:222-32. doi:10.1016/j.enpol.2015.03.012.

[11] Torriti J, Hassan MG, Leach M. Demand response experience in Europe: Policies, programmes and implementation. Energy 2010;35:1575-83. doi:10.1016/j.energy.2009.05.021.

[12] Khan AA, Razzaq S, Khan A, Khursheed F, Owais. HEMSs and enabled demand response in electricity market: An overview. Renew Sustain Energy Rev 2015;42:773-85. doi:10.1016/j.rser.2014.10.045.

[13] Cappers P, MacDonald J, Goldman C, Ma O. An assessment of market and policy barriers for demand response providing ancillary services in U.S. electricity markets. Energy Policy 2013;62:1031-9. doi:10.1016/j.enpol.2013.08.003.

[14] Vafeas A, Madina C. Developing aggregation business models : First application in the real cases Deliverable D9. 2008.

[15] Güngör VC, Sahin D, Kocak T, Ergüt S, Buccella C, Cecati C, et al. Smart Grid Technologies : Communication Technologies and Standards. IEEE Trans Ind Informatics 2011;7:529-39. doi:10.1109/TII.2011.2166794. 
[16] Kim J-H, Shcherbakova A. Common failures of demand response. Energy 2011;36:873-80. doi:10.1016/j.energy.2010.12.027.

[17] Nolan S, O'Malley M. Challenges and barriers to demand response deployment and evaluation. Appl Energy 2015;152:1-10. doi:10.1016/j.apenergy.2015.04.083.

[18] Behrangrad M. A review of demand side management business models in the electricity market. Renew Sustain Energy Rev 2015;47:270-83. doi:10.1016/j.rser.2015.03.033.

[19] Bradley P, Coke A, Leach M. Financial incentive approaches for reducing peak electricity demand, experience from pilot trials with a UK energy provider. Energy Policy 2016;98:108-20. doi:10.1016/j.enpol.2016.07.022.

[20] Haider HT, See OH, Elmenreich W. A review of residential demand response of smart grid. Renew Sustain Energy Rev 2016;59:166-78. doi:10.1016/j.rser.2016.01.016.

[21] Jaffe AB, Stavins RN. The energy paradox and the diffusion of conservation technology. Resour Energy Econ 1994;16:91-122. doi:10.1016/0928-7655(94)90001-9.

[22] Sorrell S. Understanding barriers to energy efficiency. Econ. Energy Effic., Cheltenham: Edward Elgar; 2004, p. 11-62.

[23] Gyamfi S, Krumdieck S, Urmee T. Residential peak electricity demand response-Highlights of some behavioural issues. Renew Sustain Energy Rev 2013;25:71-7. doi:10.1016/j.rser.2013.04.006.

[24] Frederiks E, Stenner K, Hobman E. Household energy use: Applying behavioural economics to understand consumer decision-making and behaviour. Renew Sustain Energy Rev 2015;41:1385-94. doi:10.1016/j.rser.2014.09.026.

[25] Sorrell S. Reducing energy demand: A review of issues, challenges and approaches. Renew Sustain Energy Rev 2015;47:74-82. doi:10.1016/j.rser.2015.03.002.

[26] Sorrell S. The economics of energy efficiency: barriers to cost-effective investment. Cheltenham: Cheltenham : Edward Elgar; 2004.

[27] Hirst E, Brown M. Closing the efficiency gap: barriers to the efficient use of energy. Resour Conserv Recycl 1990;3:267-81. doi:10.1016/0921-3449(90)90023-W.

[28] Brown MA. Market Failures and Barriers as a Basis for Clean Energy Policies. Energy Policy 2001;29:1197-207. doi:10.1016/S0301-4215(01)00067-2.

[29] Thollander P, Palm J, Rohdin P. Categorizing Barriers to Energy Efficiency : An Interdisciplinary Perspective. Energy Effic 2010:49-63. doi:10.5772/266.

[30] Chai KH, Yeo C. Overcoming energy efficiency barriers through systems approach-A conceptual framework. Energy Policy 2012;46:460-72. doi:10.1016/j.enpol.2012.04.012.

[31] Nguyen DT, Negnevitsky M, Groot M De. Pool-Based Demand Response Exchange - Concept and Modeling. IEEE Trans Power Syst 2011;26:1677-85. doi:10.1109/TPWRS.2010.2095890.

[32] Brubaker ER. Free Ride, Free Revelation, or Golden Rule? J Law Econ 1975;18:147-61.

[33] Haring TW, Kirschen DS, Andersson G. Incentive Compatible Imbalance Settlement. IEEE Trans Power Syst 2015;30:3338-46. doi:10.1109/TPWRS.2014.2387947.

[34] He X, Keyaerts N, Azevedo I, Meeus L, Hancher L, Glachant J-M. How to engage consumers in demand response: A contract perspective. Util Policy 2013;27:108-22. doi:10.1016/j.jup.2013.10.001.

[35] Satchwell A, Cappers P. A Framework for Organizing Electric Utility Regulatory and Business Models. Electr J 2015;28:119-29. doi:10.1016/j.tej.2015.09.009.

[36] Cordella A. Transaction costs and information systems: Does IT add up? J Inf Technol 2006;21:195-202. doi:10.1057/palgrave.jit.2000066.

[37] O'Sullivan J, Rogers A, Flynn D, Smith P, Mullane A, O'Malley M. Studying the maximum instantaneous non-synchronous generation in an Island system-frequency stability challenges in Ireland. IEEE Trans 
Power Syst 2014;29:2943-51. doi:10.1109/TPWRS.2014.2316974.

[38] Teng F, Aunedi M, Strbac G. Benefits of flexibility from smart electrified transportation and heating in the future UK electricity system. Appl Energy 2015. doi:10.1016/j.apenergy.2015.10.028.

[39] Martínez-Ceseña EA, Mancarella P. Distribution Network Reinforcement Planning Considering Demand Response Support. Proceeding 18th Power Syst Comput Conf 2014. doi:10.1109/PSCC.2014.7038347.

[40] Bowles S. Microeconomics: behaviour, institutions, and evolution. 3rd ed. Princeton University Press; 2004.

[41] Darby SJ, McKenna E. Social implications of residential demand response in cool temperate climates. Energy Policy 2012;49:759-69. doi:10.1016/j.enpol.2012.07.026.

[42] Parkhill K, Demski C, Butler C, Spence A, Pidgeon N. Transforming the UK Energy System: Public values, attitudes and acceptability Synthesis Report. 2013.

[43] McKenna E, Richardson I, Thomson M. Smart meter data: Balancing consumer privacy concerns with legitimate applications. Energy Policy 2012;41:807-14. doi:10.1016/j.enpol.2011.11.049.

[44] Mander S, Abi Ghanem D, Belhomme R, Delgado Espinos I, Gonzalez Sainz-Maza R, Kessels K, et al. Socio-Economic aspects of Demand Response. In: Losi A, Mancarella P, Vicino A, editors. Integr. demand response into Electr. Chain challenges, Oppor. Smart Grid Solut., Wiley-ISTE; 2015, p. 215-40.

[45] Balta-Ozkan N, Davidson R, Bicket M, Whitmarsh L. Social barriers to the adoption of smart homes. Energy Policy 2013;63:363-74. doi:10.1016/j.enpol.2013.08.043.

[46] Zhu M. Searching and switching across markets: Is consumer "inertia" the result of a mistake or a preference? In: Mehta J, editor. Behav. Econ. Compet. Consum. policy, Norwich: 2013, p. 118.

[47] European Commission. A strategy for smart, sustainable and inclusive growth. 2014. doi:10.1007/s13398-014-0173-7.2.

[48] Hart DG. Using AMI to realize the Smart Grid. IEEE Power Energy Soc. Gen. Meet., 2008, p. 1-2. doi:10.1109/PES.2008.4596961.

[49] Bera S., Misra S., Rodrigues JJPC. Cloud Computing Applications for Smart Grid: A Survey. IEEE Trans Parallel Distrib Syst 2015;26:1477-94. doi:10.1109/TPDS.2014.2321378.

[50] Muratori M, Schuelke-Leech BA, Rizzoni G. Role of residential demand response in modern electricity markets. Renew Sustain Energy Rev 2014;33:546-53. doi:10.1016/j.rser.2014.02.027.

[51] Römer B, Reichhart P, Kranz J, Picot A. The role of smart metering and decentralized electricity storage for smart grids: The importance of positive externalities. Energy Policy 2012;50:486-95. doi:10.1016/j.enpol.2012.07.047.

[52] Goldberg ML, Kennedy Agnew G. Measurement and Verification for Demand Response. 2013.

[53] Schneider Electric. Energy consumption reduction and peak- power demand response ( DR ) achieved without impact to occupant comfort. 2013.

[54] Cui B, Wang S, Xue X. Effects and performance of a demand response strategy for active and passive building cold storage. Energy Procedia 2014;61:564-7. doi:10.1016/j.egypro.2014.11.1171.

[55] Date J, Athienitis AK, Fournier M. A study of temperature set point strategies for peak power reduction in residential buildings. Energy Procedia 2015;78:2130-5. doi:10.1016/j.egypro.2015.11.289.

[56] Good N, Karangelos E, Navarro-Espinosa A, Mancarella P. Optimization under uncertainty of thermal storage based flexible demand response with quantification of residential users' discomfort. IEEE Trans Smart Grid 2015;6:2333-42. doi:10.1109/TSG.2015.2399974.

[57] European Commission. Standardisation. Digit Agenda Eur 2015. https://ec.europa.eu/digitalagenda/en/standardisation (accessed December 22, 2015).

[58] Postscapes. IoT Alliances round-up 2015. http://postscapes.com/internet-of-things-alliances-roundup (accessed March 1, 2015). 
[59] Bradshaw D, Folco G, Cattaneo G, Kolding M. Quantitative Estimates of the Demand for Cloud Computing in Europe and the Likely Barriers to Take-up. 2012.

[60] Miorandi D, Sicari S, De Pellegrini F, Chlamtac I. Internet of things: Vision, applications and research challenges. Ad Hoc Networks 2012;10:1497-516. doi:10.1016/j.adhoc.2012.02.016.

[61] Farhangi H. The Path of the Smart Grid. IEEE Power Energy Mag, 2010:18-28,. doi:10.1109/MPE.2009.934876.

[62] Ashby WR. An inroduction to cybernetics. New York: J Wiley; 1956.

[63] Ashby WR. Principles of the Self-Organizing Dynamic System. Trans Univ Illinois Symp 1962:255-78. doi:10.1080/00221309.1947.9918144.

[64] Carrez F, Bauer M, Boussard M, Bui N, Jardak C, De Loof J, et al. Internet of Things - Architecture loT A Final architectural reference model for the loT v3.0. 2013.

[65] Cisco. Building the Internet of Things 2013.

[66] ITU-T. Series Y: Global Information Infrastructure, Internet Protocol Aspects and Next-Generation Networks. 2012.

[67] Intel. Intel loT Reference Model n.d. http://download.intel.com/newsroom/kits/iot/insights/2014/gallery/images/INTEL_04_iot-01-1-01.jpg (accessed December 22, 2015).

[68] Open Interconnect Consortium. OIC Core Candidate Specification Project B. 2015.

[69] Industrial Internet Consortium. Industrial Internet Reference Architecture n.d. http://www.iiconsortium.org/IIRA.htm.

[70] Zawadzki K. Data science skill-set explained. MarketingDistillery.com 2014.

[71] European Commission. Study on the effective integration of demand energy recourses for providing flexibility to the electricity system. 2014.

[72] Xenias D, Axon CJ, Whitmarsh L, Connor PM, Balta-Ozkan N, Spence A. UK smart grid development: An expert assessment of the benefits, pitfalls and functions. Renew Energy 2015;81:89-102. doi:10.1016/j.renene.2015.03.016.

[73] Dievel P Van, Vos K De, Belmans R. Demand Response in Electricity Distribution Grids : Regulatory Framework and Barriers. Eur. Enegy Mark., 2014. doi:10.1109/EEM.2014.6861286.

[74] Good N, Martínez-Ceseña EA, Zhang L, Mancarella P. Techno-Economic and Business Case Assessment of Low Carbon Technologies in Distributed Multi-Energy Systems. Appl Energy 2016;167:158-72. doi:10.1016/j.apenergy.2015.09.089.

[75] Good N, Martínez-Ceseña EA, Mancarella P. Business cases. In: Monti A, Mancarella P, Kouramas K, Pesch D, Ellis K, editors. Energy Posit. neighborhoods smart energy Dist. methods, tools Exp. from F., Elsevier; 2016.

[76] Faruqui A, Sergici S, Sharif A. The impact of informational feedback on energy consumption-A survey of the experimental evidence. Energy 2010;35:1598-608. doi:10.1016/j.energy.2009.07.042.

[77] Nguyen DT, Negnevitsky M, Groot M De. Market-Based Demand Response Scheduling in a Deregulated Environment. IEEE Trans Smart Grid 2013;4:1948-56. doi:10.1109/TSG.2013.2258410.

[78] Cartwright E. Behavioral economics. London; New York, NY, NY: Routledge; 2011.

[79] ENA. Demand Side Response Shared Services Framework Concept Paper. 2014.

[80] Martínez-Ceseña EA, Mancarella P. Capacity to Customers ( C2C ) Development of Cost Benefit Analysis methodology for network expansion planning considering C2C interventions. 2014.

[81] Patti E, Syrri ALA, Jahn M, Mancarella P, Acquaviva A, Macii E. Distributed Software Infrastructure for General Purpose Services in Smart Grid. IEEE Trans Smart Grid, 2016; In Press. 
doi:10.1109/TSG.2014.2375197.

[82] Dowson M, Poole A, Harrison D, Susman G. Domestic UK retrofit challenge: Barriers, incentives and current performance leading into the Green Deal. Energy Policy 2012;50:294-305.

doi:10.1016/j.enpol.2012.07.019.

[83] DECC. Planning our electric future: a White Paper for secure, affordable and low carbon electricity 2011.

[84] Schachter JA, Mancarella P. Demand Response Contracts as Real Options: A Probabilistic Evaluation Framework Under Short-Term and Long-Term Uncertainties. IEEE Trans Smart Grid 2015;In Press. doi:10.1109/TSG.2015.2405673.

[85] Martínez-Ceseña EA, Capuder T, Mancarella P. Flexible Distributed Multienergy Generation System Expansion Planning Under Uncertainty. IEEE Trans Smart Grid 2015;In Press:348-57. doi:0.1109/TSG.2015.2411392.

[86] Williamson OE. Markets and Hierarchies: Analysis and Antitrust Implications : a Study in the Economics of Internal Organization. Free Press; 1983.

[87] Althaher S, Mancarella P, Mutale J. Automated Demand Response From Home Energy Management System Under Dynamic Pricing and Power and Comfort Constraints. IEEE Trans Smart Grid 2015;6:1874-83. doi:10.1109/TSG.2014.2388357.

[88] Mert W, Suschek-Berger J, Tritthart W. Consumer Acceptance of Smart Appliances - D5.5 Smart-A project. 2008.

[89] Darby S. Smart metering: what potential for householder engagement? Build Res Inf 2010;38:442-57. doi:10.1080/09613218.2010.492660.

[90] Naesje PC, Andersen TK, Saele H. Customer response on price incentives. ECEEE 2005 Summer Study 'Energy Savings What Work. Who Deliv., vol. 3, 2005, p. 1259-1269.

[91] Shou L, Shang X, Chen K, Chen G, Zhang C. Supporting Pattern-Preserving Anonymization for TimeSeries Data. IEEE Trans Knowl Data Eng 2013;25:877-92. doi:10.1109/TKDE.2011.249.

[92] DECC. Smart Meters, Great Britain , Quarterly report to end June 2014. 2014.

[93] European Commission. Benchmarking smart metering deployment in the EU-27 with a focus on electricity. Brussels: 2014.

[94] OGC. Sensor Model Language (SensorML) n.d. http://www.opengeospatial.org/standards/sensorml (accessed December 23, 2015).

[95] Compton M, Barnaghi P, Bermudez L, García-Castro R, Corcho O, Cox S, et al. The SSN ontology of the W3C semantic sensor network incubator group. J Web Semant 2012;17:25-32. doi:10.1016/j.websem.2012.05.003.

[96] Kno.e.sis. Semantic Sensor Web n.d. http://knoesis.org/projects/ssw (accessed December 23, 2015).

[97] Moore G. Cramming More Components Onto Integrated Circuits. Electronics 1965:114-7. doi:10.1109/JPROC.1998.658762.

[98] Mittal S, Ruth M, Pratt A, Lunacek M, Krishnamurthy D, Jones W. A System-of-Systems Approach for Integrated Energy Systems Modeling and Simulation Preprint. 2015.

[99] Ellis K, Cheevers P. Report on Refined Specification for Neighbourhood Management Architecture. 2014.

[100] OpenHAB. openHAB n.d. http://www.openhab.org/ (accessed December 23, 2015).

[101] Open Interconnect Consortium. IoTivity n.d. https://www.iotivity.org/ (accessed December 22, 2015).

[102] The Open Data Foundation. The Open Data Foundation n.d. http://www.opendatafoundation.org/ (accessed December 22, 2015). 
[103] W3C. Linked Data 2015. http://linkeddata.org/ (accessed October 26, 2015).

[104] Zheng L, Lu N, Cai L. Reliable Wireless Communication Networks for Demand Response Control. IEEE Trans Smart Grid 2013;4:133-40. doi:10.1109/TSG.2012.2224892.

[105] Aust S, Prasad RV, Niemegeers IGMM. Performance evaluation of Sub $1 \mathrm{GHz}$ wireless sensor networks for the smart grid. 37th Annu. IEEE Conf. Local Comput. Networks, 2012, p. 292-5. doi:10.1109/LCN.2012.6423632.

[106] European Commission. Energy efficiency vocabularies and ontologies. Proc. 4th Work. organised by EEB Data Model. Community ICT Sustain. Places, 2013. doi:10.2759/40897.

[107] den Hartog F, Daniele L, Roes J. Study on Semantic Assets for Smart Appliances Interoperability: First interim report. 2014.

[108] Daniele L, den Hartog F, Roes J. Study on Semantic Assets for Smart Appliances Interoperability: Third interim report. 2014.

[109] International B. building SMART: International home of openBIM n.d. http://www.buildingsmarttech.org/ (accessed December 22, 2015).

[110] Dice, The Linux Foundation. 2013 Linux Jobs Report. 2013.

[111] Lonoff Schiff J. 8 Tips for Retaining Top IT Talent. CIO.com 2013.

[112] Flamm A, Scott D. Electricity Balancing Significant Code Review - Final Policy Decision. 2014.

[113] Ofgem. RIIO: A new way to regulate energy networks. 2010.

[114] Enernoc. The Demand Response Baseline: White Paper. 2008.

[115] Papadaskalopoulos D, Pudjianto D, Strbac G. Decentralized Coordination of Microgrids With Flexible Demand and Energy Storage. IEEE Trans Sustain Energy 2014;PP:1-1. doi:10.1109/TSTE.2014.2311499.

[116] Mohsenian-Rad A-H, Wong VWS, Jatskevich J, Schober R, Leon-garcia A. Autonomous Demand-Side Management Based on Game-Theoretic Energy Consumption Scheduling for the Future Smart Grid. IEEE Trans Smart Grid 2010;1:320-31.

[117] Alnaser SW, Ochoa LF. Advanced Network Management Systems: A Risk-Based AC OPF Approach. Power Syst IEEE Trans 2015;30:409-18. doi:10.1109/TPWRS.2014.2320901.

[118] Fong C. Social preferences, self-interest, and the demand for redistribution. J Public Econ 2001;82:22546. doi:10.1016/S0047-2727(00)00141-9.

[119] Vespignani A. Modelling dynamical processes in complex socio-technical systems. Nat Phys 2011;8:329. doi:10.1038/nphys2160.

[120] Bale CSE, Varga L, Foxon TJ. Energy and complexity: New ways forward. Appl Energy 2015;138:150-9. doi:10.1016/j.apenergy.2014.10.057.

[121] Pfenninger S, Hawkes A, Keirstead J. Energy systems modeling for twenty-first century energy challenges. Renew Sustain Energy Rev 2014;33:74-86. doi:10.1016/j.rser.2014.02.003.

[122] Daniell R. Creating the right environment for demand- side response : next steps. 2013.

[123] Gabriel SA, Conejo AJ, Plazas MA, Balakrishnan S. Optimal price and quantity determination for retail electric power contracts. IEEE Trans Power Syst 2006;21:180-7. doi:10.1109/TPWRS.2005.860920.

[124] Yoon KH, Ratti RA. Energy price uncertainty, energy intensity and firm investment. Energy Econ 2011;33:67-78. doi:10.1016/j.eneco.2010.04.011.

[125] Sorrell S. The economics of energy service contracts. Energy Policy 2007;35:507-21. doi:10.1016/j.enpol.2005.12.009.

[126] Strbac G, Aunedi M, Pudjianto D, Teng F, Djapic P, Druce R, et al. Value of Flexibility in a Decarbonised Grid and System Externalities of Low-Carbon Generation Technologies. 2015. 
doi:10.13140/RG.2.1.2336.0724.

[127] Poudineh R, Jamasb T. Distributed generation, storage, demand response and energy efficiency as alternatives to grid capacity enhancement. Energy Policy 2014;67:222-31. doi:10.1016/j.enpol.2013.11.073.

[128] Arthur BW. Increasing returns and the new world of business. Harv Bus Rev 1994;74:100-9. 